\title{
Quarterly Progress Report Covering Period July 1 Through September 29, 1978- The Study of Plutonium Oxide Leak Rates from Shipping Containers
}

November 1978

Prepared for the U.S. Nuclear Regulatory Commission under a Related Services Agreement with the U.S. Department of Energy

Contract EY-76-C-06-1830

Fin No. B2093

Pacific Northwest Laboratory Operated for the U.S. Department of Energy by Battelle Memorial Institute 
NOTICE

This report was prepared as an account of work sponsored by the United States Government. Neither the United States nor the United States Nuclear Regulatory Commission, nor any of their employees, nor any of their contractors, subcontractors, or their empioyees, makes any warranty, express or implied, or assumes any legal liability or responsibility for the accuracy, completeness or usefulness of any information, apparatus, product or process disclosed, or represents that its use would not infringe privately owned rights.

\author{
PACIFIC NORTHWEST LABORATORY \\ operated by \\ BATTELLE \\ for the \\ UNITED STATES DEPARTMENT OF ENERGY \\ Under Contract EY-76-C-06-1830
}




\section{$33679000518094 \quad$ Accession No. \\ PNL-2260-8}

INTERIM REPORT

CONTRACT PROGRAM OR PROJECT TITLE: $\mathrm{PuO}_{2}$ Particle Leak Studies

SUBJECT OF THIS DOCUMENT: QUARTERLY PROGRESS REPORT COVERING PERIOD

JULY 1 THROUGH SEPTEMBER 29, 1978 -- THE STUDY

OF PLUTONIUM OXIDE LEAK RATES FROM SHIPPING

CONTAINERS

AUTHOR(S): L.C. Schwendiman, W.J. Madia, J. Mishima, S.L. Sutter, T.J. Bander E.W. Schmidt and J.D. Yesso

TYPE OF REPORT: Status Surmary Report

DATE OF DOCUMENT: October 20, 1978

RESPONSIBLE NRC INDIVIDUAL AND NRC OFFICE OR DIVISION:

William R. Lahs

Systems Performance Branch

Div. of Safeguards, Fuel Cycle \& Environmental Research

This document was prepared primarily for preliminary or internal use. It has not received full review and approval. Since there may be substantive changes, this document should not be considered final.

PACIFIC NORTHWEST LABORATORY

Operated By

BATTELLE MEMORIAL INSTITUTE

for the

UNITED STATES DEPARTMENT OF ENERGY

Prepared for

U.S. Nuclear Regulatory Commission

Washington, D.C. 20555

Under Interagency Agreement

EY-76-C - 06-1830

NRC FIN No. B2093

INTERIM REPORT 
.

. 
TABLE OF CONTENTS

Page

INTRODUCTION

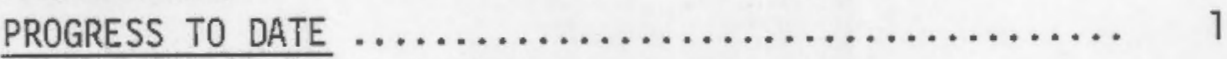

TASK A -- Review literature and theoretical work relating to transmission of particles through channels ................... 1

TASK B -- Investigate the relationship of gas flow rates, leak geometries, pressure and temperatures $\ldots \ldots \ldots \ldots \ldots \ldots \ldots \ldots \ldots, \quad$ i

TASK C -- Measure transmission of a well characterized simulant ( $\mathrm{UO}_{2}$ powder) through leaks characterized in Task $B \ldots \ldots \ldots .2$

TASK D -- Measure fuel grade $\mathrm{PuO}_{2}$ leaks through a "standard leak" incorporated into a suitable container ...................... 11

TASK E -- Investigate $\mathrm{PuO}_{2}$ leaks through simulated defected containers $. . . \ldots \ldots \ldots \ldots . . . .$.

REFERENCES

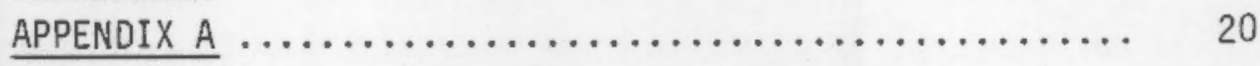

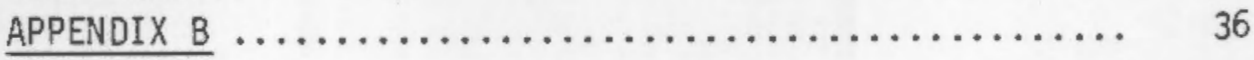




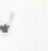

. 


\section{QUARTERLY PROGRESS REPORT \\ Ju1y 1 - September 29, 1978 \\ STUDY OF PLUTONIUM OXIDE LEAK RATES FROM SHIPPING CONTAINERS}

\section{INTRODUCTION}

This study was initiated in Dctober, 1976, as out?ined in the 189 research proposal submitted previousiy. Several tasks are to be undertaken in this study which, when combined, have the end objective of defining the leak rates of plutonium oxide powder from characterized leaks.

This is the eighth quarterly report of this work. Previous reports were issued as BNWL-2260-1, -2, $-3,-4,-5,-6$, and -7 . Each task will be identified and the progress during the reporting period briefly described.

PROGRESS TO DATE

TASK A -- Review literature and theoretical work relating to transmission of particles through channels

Task objective has been fulfilled, and two reports issued: "Supporting Information for the Estimation of Plutonium Oxide Leak Rates Through Very Saml] Apertures," by L.C. Schwendiman, BNWL-2198, and "Transport of Particles Through Gas Leaks -- A Review," by L.C. Schwendiman and S.L. Sutter, BNWL-2218, January 1977.

\section{TASK B -- Investigate the relationship of gas flow rates, leak geometries,}

Milestone 1. Review literature on topic.

Milestone 2. Report on technical 1iterature.

These milestones were reached and a document, "Estimation of Gas Leak Rates Through Very Smal1 Orifices and Channels," by H.J. Bomelburg, BNWL-2223, was issued.

Milestone 3. Select method and design apparatus for flow experiments.

Milestone 4. Fabricate and assemble apparatus.

Milestone 5. Test apparatus.

Milestone 6. Conduct first test.

Milestones 1 through 6 were completed in FY-1977.

Milestone 7. Complete test series (orifices). January 1978. 
Milestone 8. Draft report. March 1978.

Milestone 9. Issue report.

The document, "Measured Airflow Rates Through Microorifices and Flow Prediction Capabi1ity," NUREG/CR-0066 (PNL-2611) was issued in July 1978.

Milestone 10. Fabricate microcapillaries.

Milestone 11. Complete test series. April 1978.

Milestone 12. Draft report.

A revised draft of a document, "Measured Airflow Rates Through Tubing of Fine Bore," is being circulated for internal review.

TASK C -- Measure transmission of a well-characterized simulant ( $\mathrm{UO}_{2}$ powder) through leaks characterized in Task B

Milestone 1. Pressure vessel for simulating container available for experiments. June 1978.

Milestone 2. Convert airflow apparatus. Completed January 1978.

Milestone 3. First experiment completed.

Initial experiments to measure the transmission of $\mathrm{UO}_{2}$ when the leakpath is above (APLA) or below (UPL) the powder level were completed in the third quarter and further experiments continued into the fourth quarter of FY-1978.

- Leakpaths Above The Static Powder Level With Powder Agitation (APLA)

Seventy-two experiments were completed during this reporting period. Three of these experiments investigated individual orifices in pressure decay. Results of all the experiments for which the uranium measurements have been completed are shown in Table A-1 (see Appendix A). The quantity of depleted uranium dioxide (DUO) measured is reported as total $\mu \mathrm{g}$ DUO transmitted, $\mu \mathrm{g}$ DUO/min, and $\mu \mathrm{g}$ DUO/cc air.

-- Individual orifice or capillary experiments

Experiments were completed on single orifices and capillaries. The powder transmission rates $(\mu \mathrm{g} / \mathrm{min})$ continued to increase as a function of orifice diameter (Figure 1). Figure 2 plots capillary DU0 powder flow as a function of pressure for three nominal capillary diameters at two lengths. Powder transmission rate increases with diameter are mixed and appeared to increase with length, i.e., at 1000 psig, the longer $150 \mu \mathrm{m}$ diameter capillary had six times the flow the the shorter $150 \mu$ m diameter capillary. The two values for the longer 250 win diameter $(30,500$ psig) are in question because of plugging which was apparent when the experiment began. However, these data are too few to show a functional relationship. Further investigation will be 


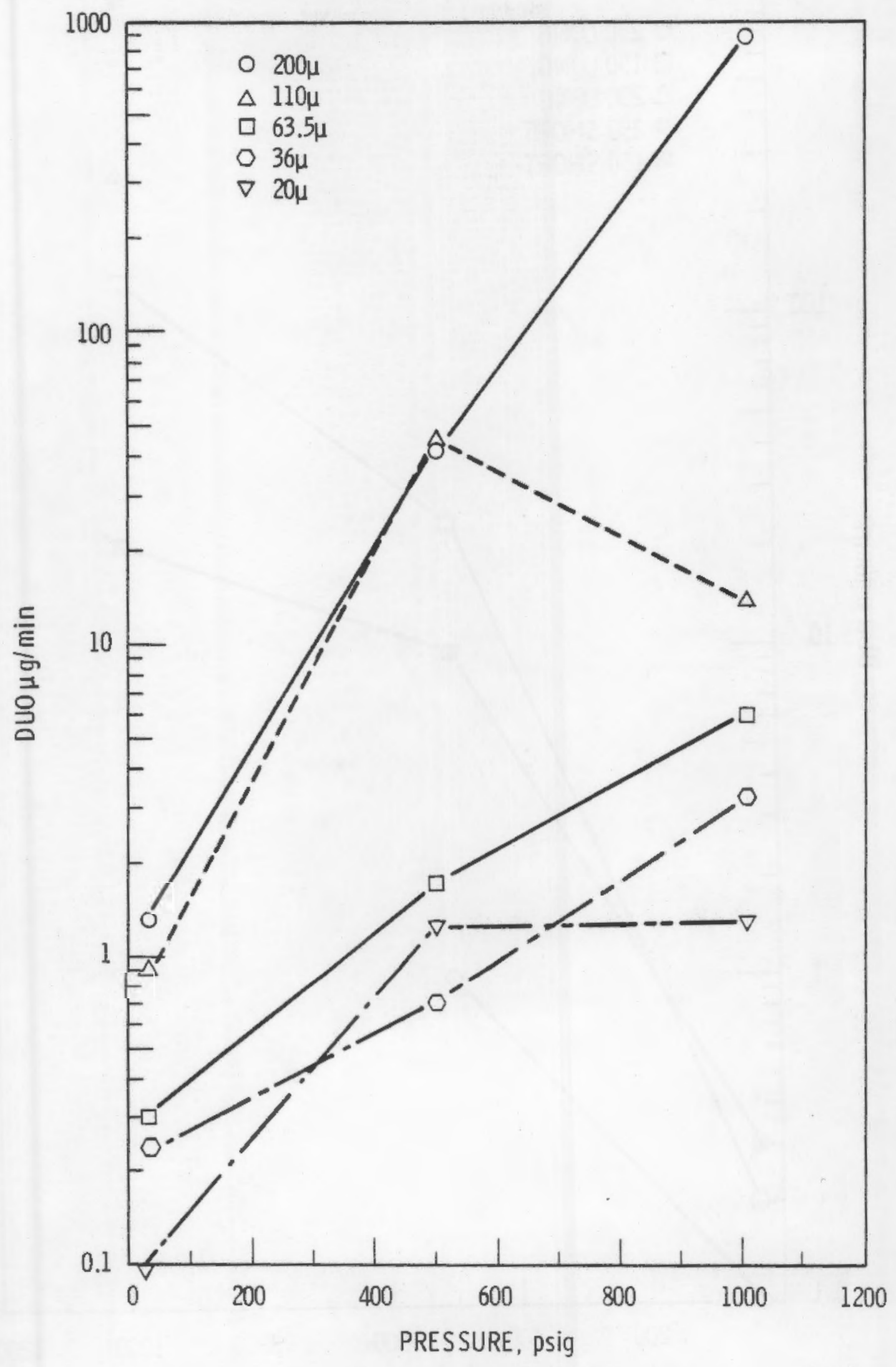

FIGURE 1 -- APLA Powder Leak Rates Through Orifices as a Function of Pressure 


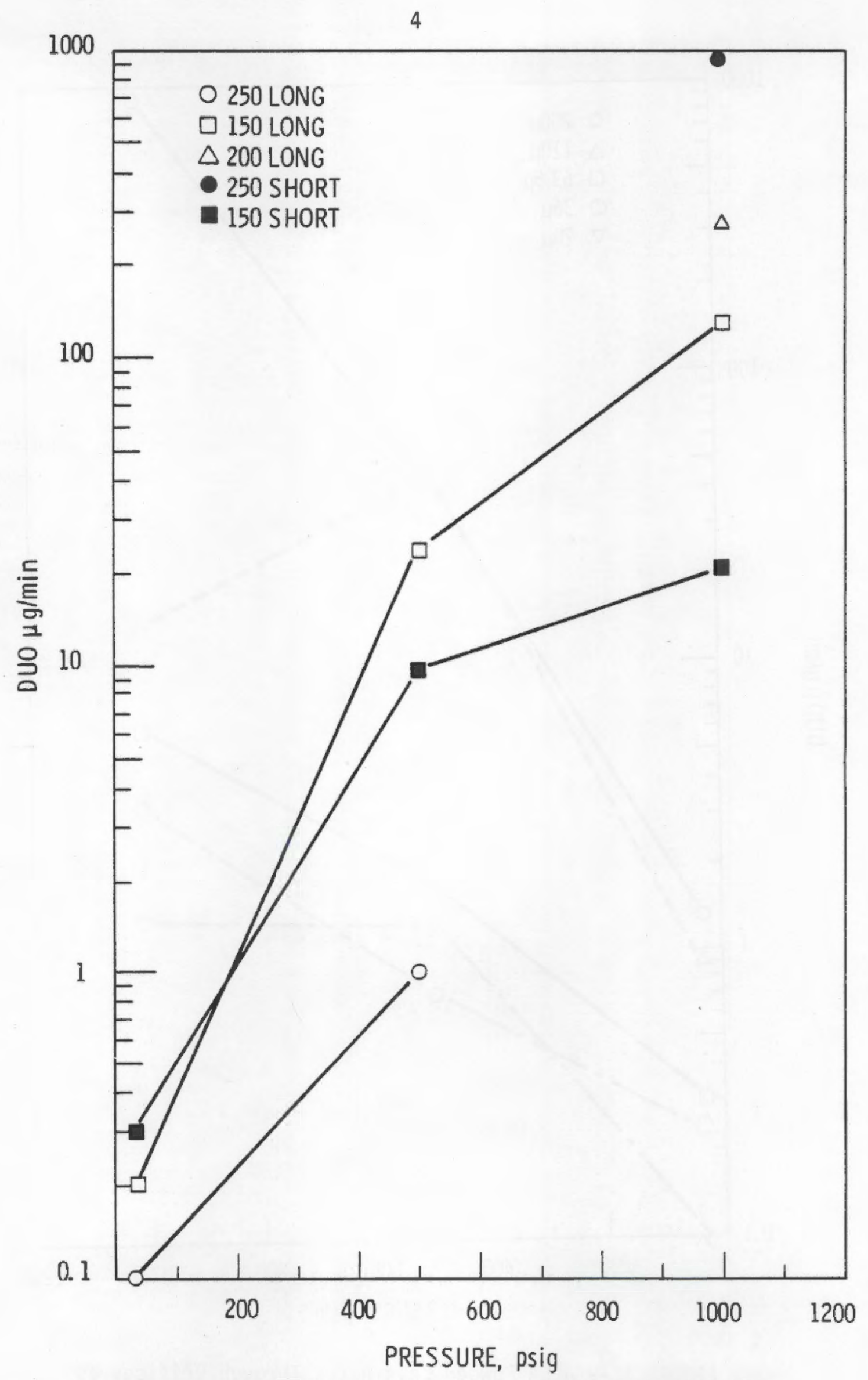

FIGURE 2 -- APLA Powder Leak Rates through Capillaries as a Function of Pressure 
completed when replacements for broken and clogged capillaries are fabricated and characterized.

In the initial experiments, the known airflow rate at the designated pressure for the orifice or capillary was maintained by a vacuum. This compensated for the buildup of back pressure that would lower the flow rate below the desired value. Currently, a series of experiments have begun which do not use a vacuum to maintain flow; as a result, orifices exhibit a flow lower than the characterized airflow rate. Table I shows the percentage of deviation from the characterized orifice flow for two pressures.

\section{TABLE I}

UNAIDED AIRFLOW AS PERCENT OF CHARACTERIZED

AIRFLOW RATE BY ORIFICE SIZE

$\begin{array}{cccccc}\frac{\text { psig }}{1000} & \frac{20 \mu \mathrm{m}}{76,12} & \frac{36 \mu \mathrm{m}}{74,7} & \frac{63.5 \mu \mathrm{m}}{28} & \frac{110 \mu \mathrm{m}}{64} & \frac{200 \mu \mathrm{m}}{84} \\ 500 & 20 & 57 & 27 & 73 & 100\end{array}$

-- Multiple orifice and capillary experiments

Experiments are being performed to assess the anticipated standard deviation for individual powder transmission rates, for leakpaths of each nominal diameter under upstream conditions, and for changes in powder transmission as a function of time.

Multiple orifice runs showed as much as 100 percent standard deviation between samples. Table II compares the deviation between samples. There tends to be better agreement between samples at higher pressures and larger orifice diameters. This is the deviation between four samples for the nominat $20 \mu \mathrm{m}$ and $36 \mu \mathrm{m}$ diameter, 3 for the $63.5 \mu \mathrm{m}$ and $110 \mu \mathrm{m}$, and 2 for the $200 \mu \mathrm{m}$.

\section{TABLE II}

VARIATION BETWEEN SAMPLES, TOTAL OUO TRANSMITTED

AS PERCENT OEVIATION, SAME AEROSOLIZEO POWDER

$\begin{array}{rccccc}\frac{\text { psig }}{1000} & \frac{20 \mu \mathrm{m}}{65} & \frac{36 \mu \mathrm{m}}{55} & \frac{63.5 \mu \mathrm{m}}{50} & \frac{110 \mu \mathrm{m}}{29} & \frac{200 \mu \mathrm{m}}{10} \\ 500 & 35 & 29 & 6 & & \\ 30 & 62 & 100 & 62 & & \end{array}$


The powder transmission does not seem to increase with time at 500 psig for the orifices tested. Fig. 3 is a bar graph comparing the total DUO transmitted for the longest sampling time of the multiple time increment experiments, the multiple uniform time samples, and the single orifice results. The 63.5 micron orifice that was sampled for 60 minutes at 1000 psig was the only one that showed a statistically significant increase in the amount of DUD transmitted above shorter time (10 min.) samples. The other orifices did not show , an increase in DUO transmission with time. The results at $30 \mathrm{psig}$ are mixed, and the potential deviation between samples make these results inconclusive.

The objective of this study is to correlate powder transmission with known gas leak rates. A finite amount of DUO powder seems to be transmitted in each $c c$ of air as shown in the piot of $\mu \mathrm{g} / \mathrm{cc}$ and airflow in Figure 4 . If each cc of air transmitted contains a finite amount of uranium, then more DUO powder will be transmitted the higher the airflow rate. Figure 5 illustrates this trend by plotting of the transmission in $\mu \mathrm{g} / \mathrm{min}$ as a function of airflow rate.

\section{-- Pressure decay experiments}

Six times as much DUO was transmitted through two nominal $63.5 \mu \mathrm{m}$ orifices in a pressure decay experiment requiring 20 hours as through a single $63.5 \mu \mathrm{m}$ orifice sampling for 10 minutes at $1000 \mathrm{psig}$. These experiments agitated the powder; the vessel was pressurized to 1000 psig and allowed to depressurize. There was no attempt to maintain a specific flow rate.

Three additional pressure decay experiments measured powder transmission under a decaying pressure regime. The estimated and required time to depressurize the vessel are shown in Table III. (The calculational methodology for the volume decay is shown in Appendix B.) The measured pressure decay is plotted in Figure 6.

TABLE III

TIME REQUIRED TO DEPRESSURIZE VESSEL

\begin{tabular}{|c|c|c|}
\hline Diameter & $\begin{array}{l}\text { Estimated } \\
\text { Time, Min. }\end{array}$ & $\begin{array}{l}\text { Required } \\
\text { Time, Min. }\end{array}$ \\
\hline 100 & 75 & 132 \\
\hline 125 & 48 & 85 \\
\hline 200 & 19 & 27 \\
\hline
\end{tabular}




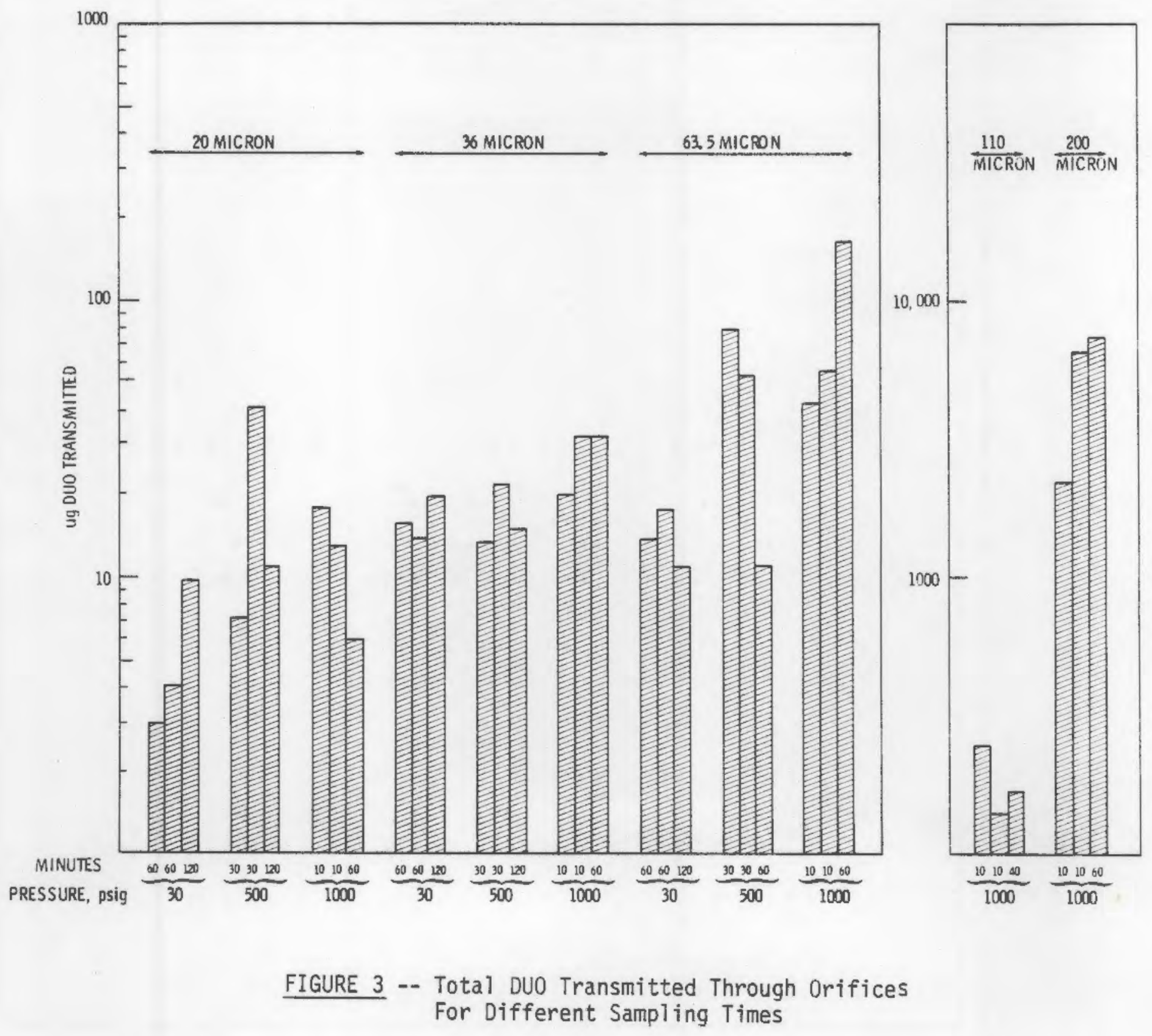




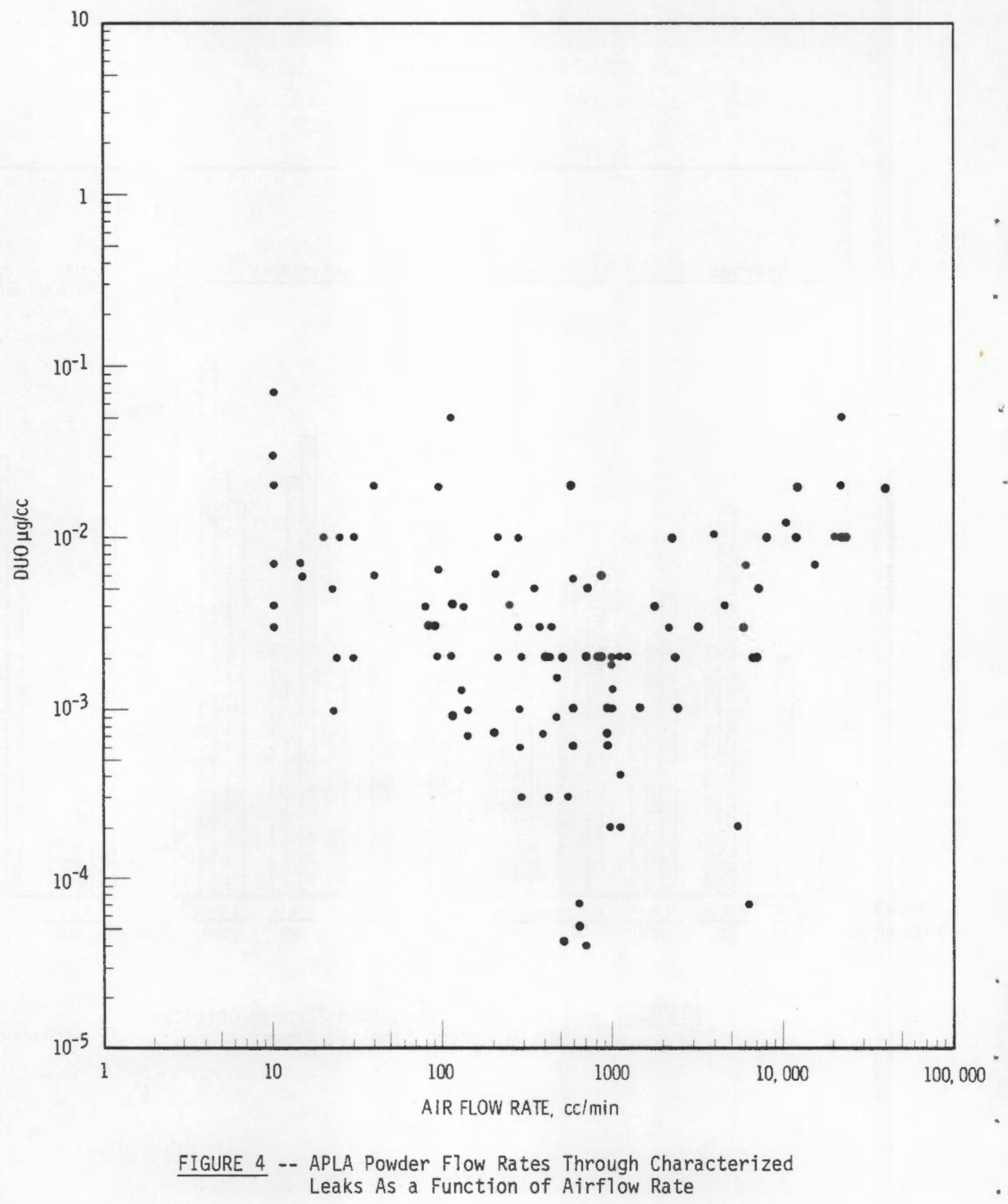




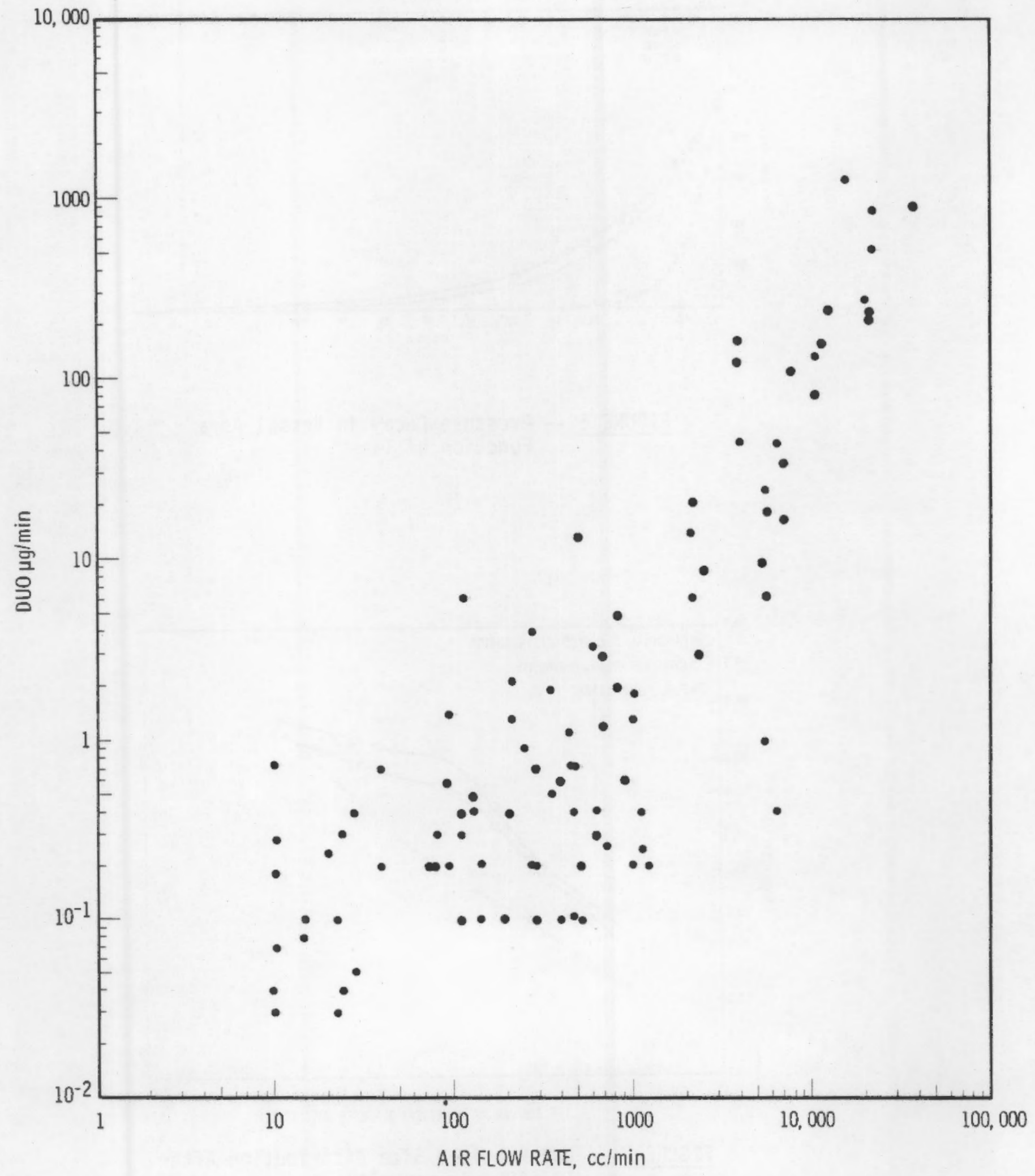

FIGURE 5 -- APLA Powder Flow Rates Through Characterized Leaks as a Function of Airflow Rates 


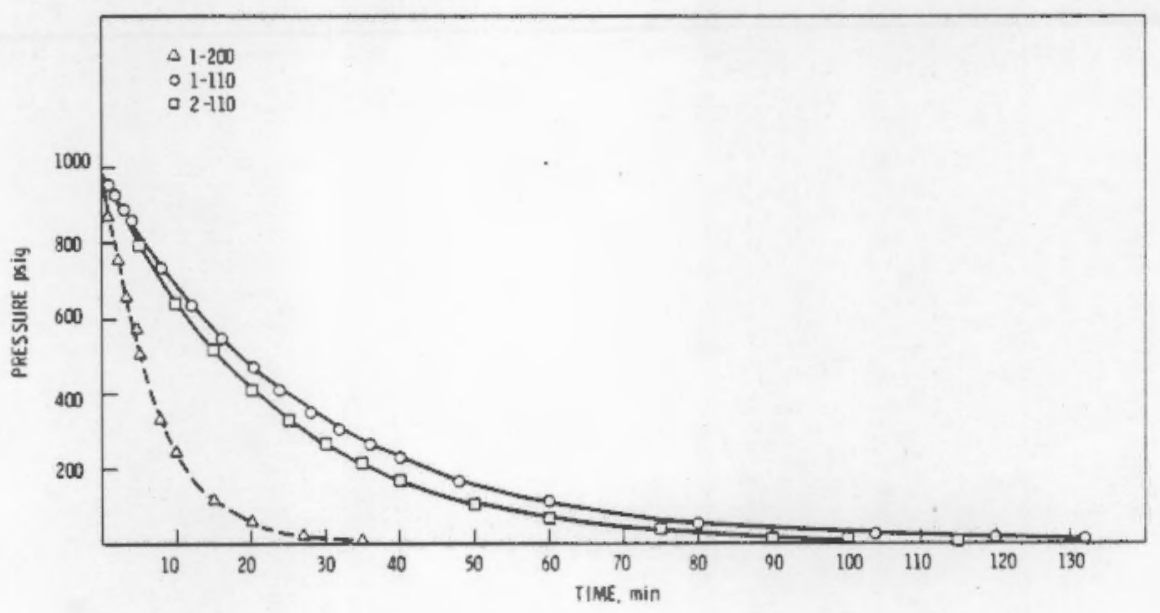

FIGURE 6 -- Pressure Decay in Vessel As a Function of Time

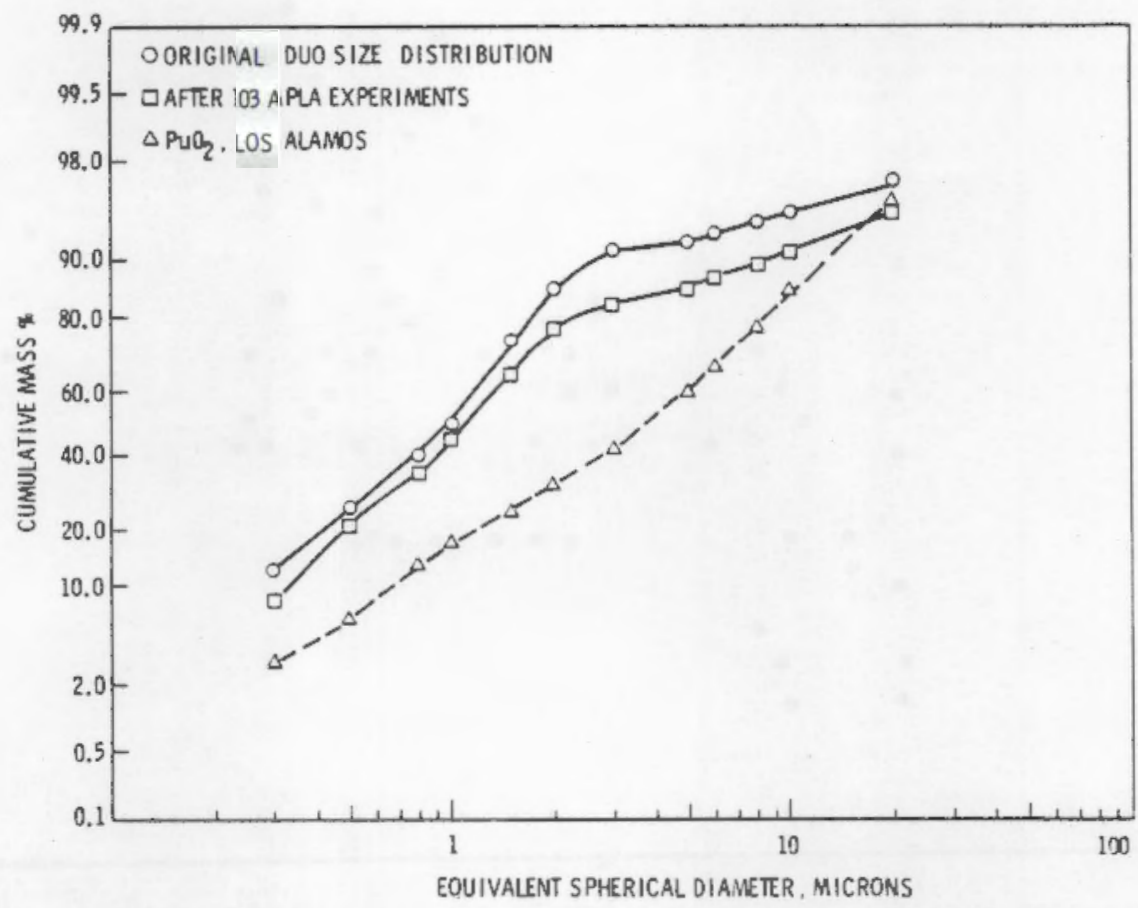

FIGURE 7 DUO Particle Size Distribution After
103 APLA Experiments 
In event of an accident, several hours could elapse before a depressurized vessel with a minute break reaches ambient pressure, with no additional pressurization.

The DUO particle size did not change significantly during the APLA experiments. Figure 7 shows the DUO particle size distribution after 103 APLA experiments using the same bulk powder. Mass median diameter is now $1.1 \mu \mathrm{m}$, and there does not seem to be a significant depletion of fine particles by the aerosolization techniques.

- Leakpath Under the Powder Level (UPL)

Measurements of the DUO transmitted through leakpaths under the powder level are tabulated in Table A-2 (see Apoendix A). The transmission rates for all nominal orifice sizes under $200 \mu \mathrm{m}$ appeared to maximize at approximately $100 \mathrm{psig}$, decrease at $500 \mathrm{psig}$, and to increase at $1000 \mathrm{psig.}$ ( 1 ) Powder transmission rates appear to increase with increasing diameter for the capillaries tested; all diameters were $182 \mu \mathrm{m}$ or greater, as shown in plots in Figures 8 and 9. Agitation did not seem to be an important parameter. As the capillary length increased, the powder flow generally decreased. An exception was the $150 \mu \mathrm{m}$ diameter at 1000 psig with no agitation, which was confirmed in reruns. This phenomenon will be investigated further when fabrition of more capillaries is completed.

TASK D -- Measure fuel grade $\mathrm{PuO}_{2}$ leaks through a "standard leak" incorporated into a suitable container

Milestone 1. Design of experimental equipment.

Milestone 2. Assembly of experimental system.

Milestone 3. Simulant experiments.

Milestone 4. Transfer to glovebox.

Milestone 5. Conduct "hot experiments".

- "Standard Leak" Configuration

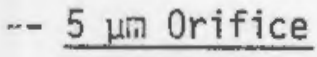

As discussed in the June 19, 1978, proposal for extended work (Proposal No. 585-J-4069R), the data at the bounding limits of each parameter must be adequately measured in order to determine the effects of the parametric interactions. Furthermore, to determine whether the relationships are 1 inear or curvilinear, an internal data point must also be measured. Based on 


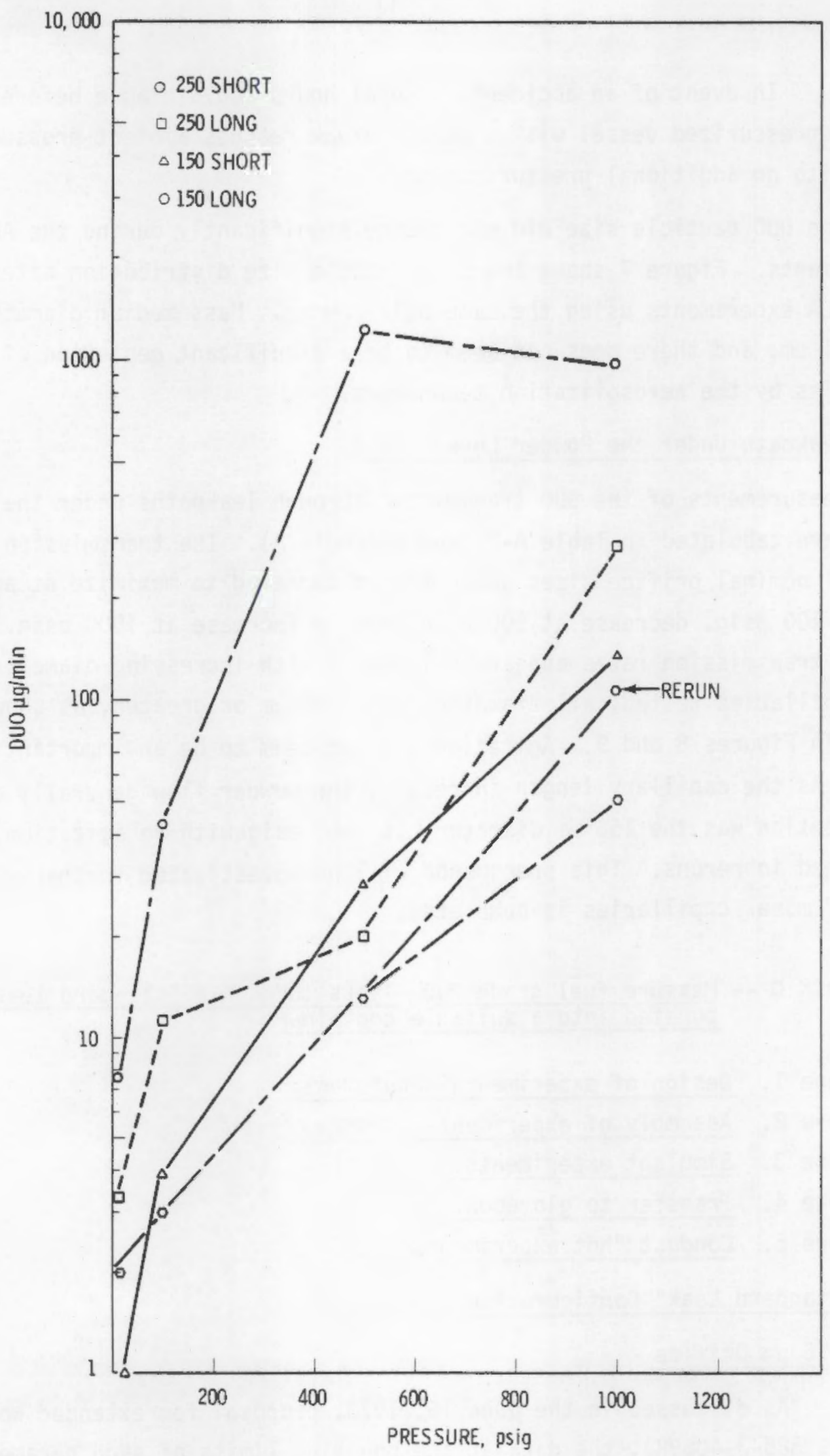

FIGURE 8 -- UPL Powder Leak Rates Through Capillaries As a Function of Pressure (Agitated) 


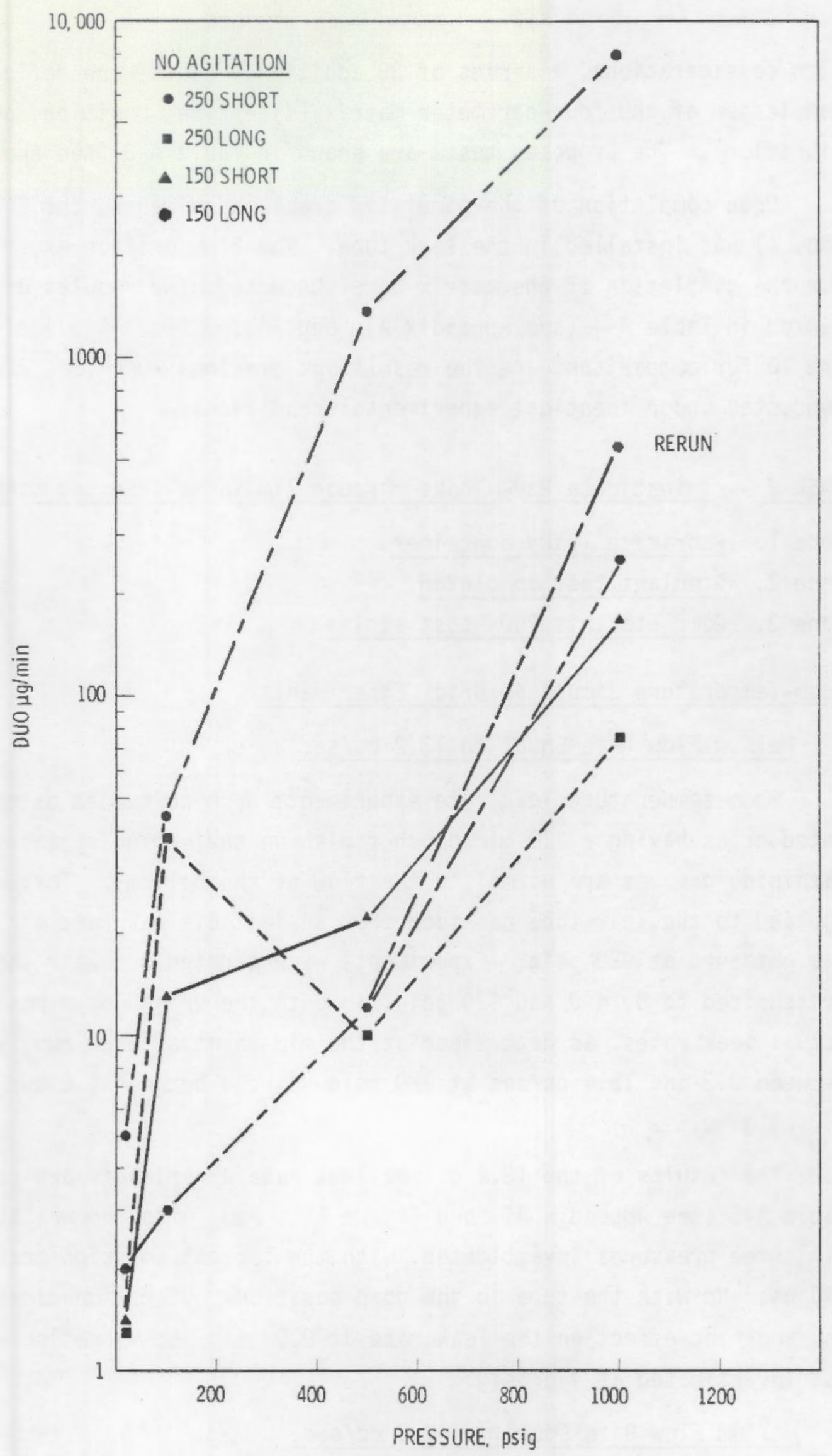

FIGURE 9 -- UPL Powder Leak Rates Through Capillaries As a Function of Pressure (No Agitation) 
such considerations, a series of 38 additional tests were defined for the completion of the four-parameter matrix (size/type, position, pressure, vibration). The proposed tests are shown in Table A-3 (see Appendix A).

Upon completion of the simulated crack experiments, the $5 \mu \mathrm{m}$ orifice (No. 4) was installed in the leak tube. The $5 \mu \mathrm{m}$ orifice experiments required for the completion of the matrix were conducted, the results of which are presented in Table A-4 (see Appendix A) and Figure 10. Also included in Figure 10 for comparison, are the results of previous runs $(28,28 a, 33,33 a)$ conducted under identical experimental conditions.

\section{$\underline{\text { TASK E -- Investigate } \mathrm{Pu}_{2} \text { leaks through simulated defected containers }}$}

Milestone 1. Fabricate leaky container.

Milestone 2. Simulant test completed.

Milestone 3. Complete this $\mathrm{PuO}_{2}$ test series.

- Room-Temperature Simulated Crack Experiments

-- Helium Flow Rate Equal to $13.2 \mathrm{cc} / \mathrm{sec}$

Room-temperature leak rate experiments were conducted using the simulated crack having a 220 -microinch finish on the mating surfaces (the machining grooves are normal to the flow of the helium). Torque was applied to the leak tube cap such that an initial leak rate of $13.2 \mathrm{cc} / \mathrm{sec} \mathrm{He}$ was obtained at 920 psig. Experiments were carried out with the leak tube pressurized to 0,440 and $920 \mathrm{psig}$, in both the up and down positions. The actual leak rates, as determined at the mid-point of each run, varied between 9.3 and $12.4 \mathrm{cc} / \mathrm{sec}$ at $920 \mathrm{psig} \mathrm{He}$, and between 2.5 and $3.0 \mathrm{cc} / \mathrm{sec}$ at 440 psig He.

The results of the $13.2 \mathrm{cc} / \mathrm{sec}$ leak rate experiments are presented in Table A-5 (see Appendix A) and Figure 11. $\mathrm{PuO}_{2}$ emission was observed at a11 three pressures investigated, with the largest emission occurring at $440 \mathrm{psig}$ He with the tube in the down position. Vibration did not have any apparent effect on the leak rate at 920 psig He; vibration effects were not investigated at 440 psig.

-- Hel ium Flow Rate Equal to $17.3 \mathrm{cc} / \mathrm{sec}$.

In these experiments, torque was applied to the leak tube cap to provide an initial leak rate of $17.3 \mathrm{cc} / \mathrm{sec}$ at $920 \mathrm{psig}$ He. Actual leak rates 


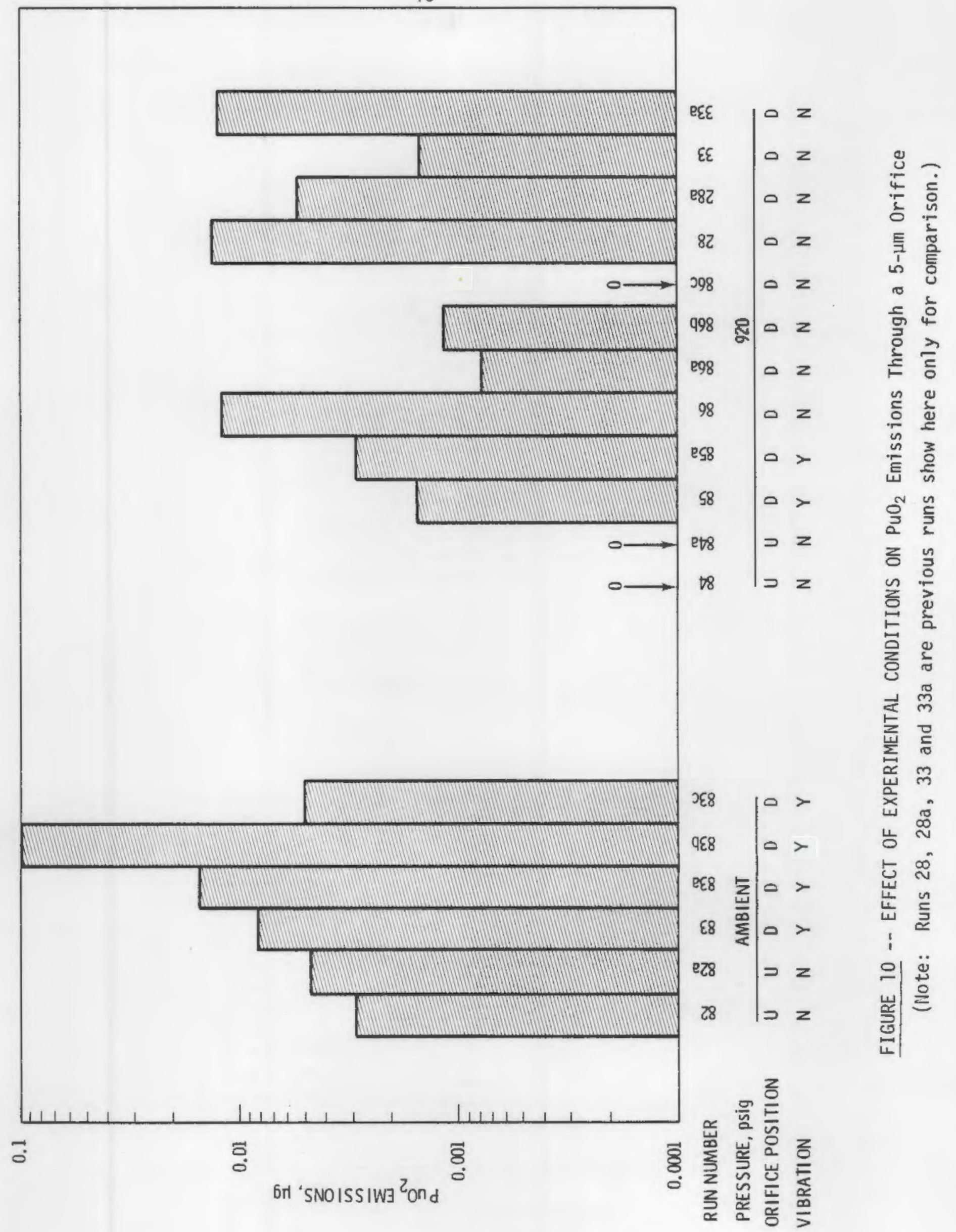




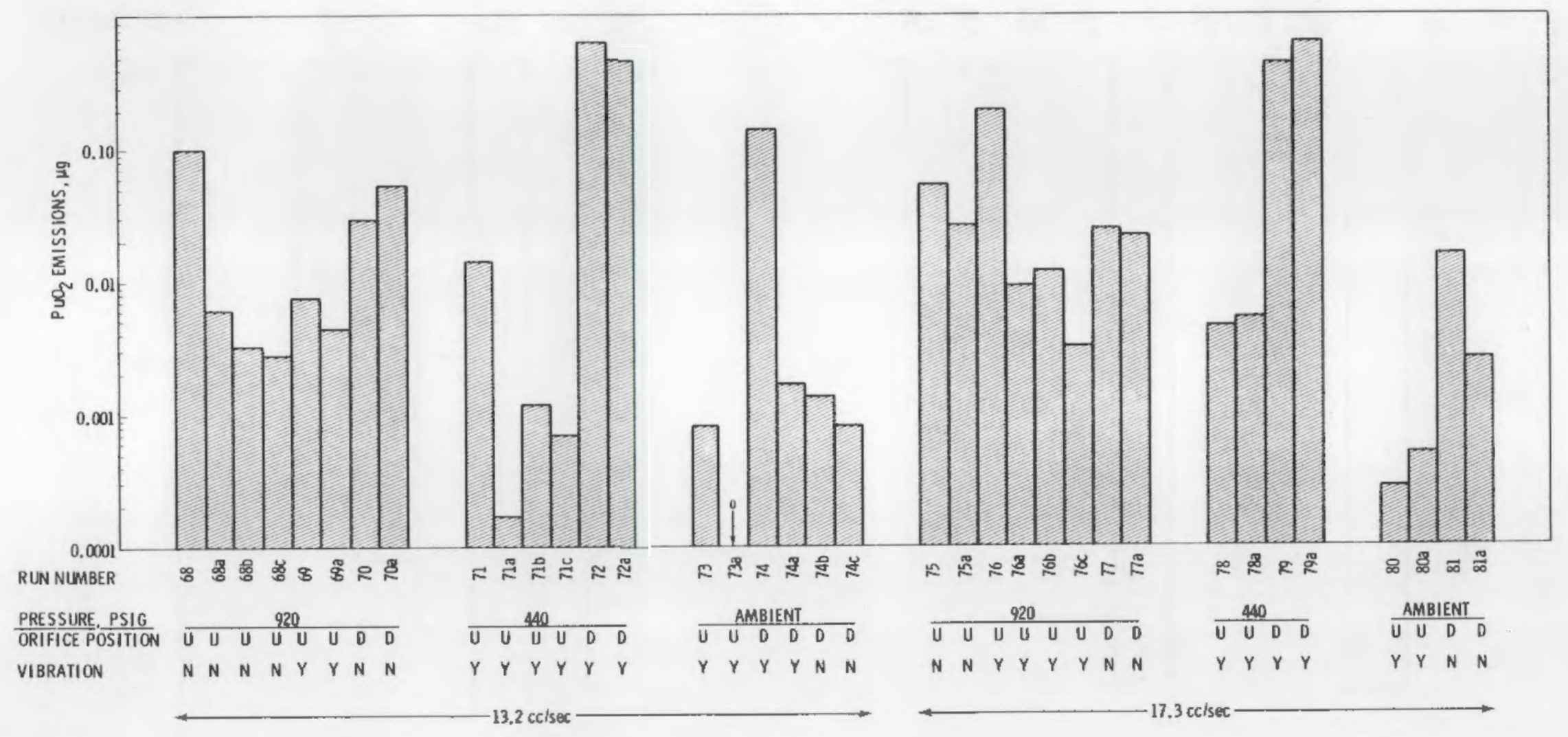

FIGURE 11 -- Effect of Experimental Conditions on $\mathrm{PuO}_{2}$ Emissions through A Simulated Crack at Room Temperature With Two Initial He Flow Rates 
varied from 8.25 to $16.3 \mathrm{cc} / \mathrm{sec}$ at $920 \mathrm{psig}$, but were a consistent 2.6 $\mathrm{cc} / \mathrm{sec}$ at $440 \mathrm{psig}$. Consistent with observations at 13.2 and $9.8 \mathrm{cc} / \mathrm{sec}$, the largest $\mathrm{PuO}_{2}$ emission occurred at $440 \mathrm{psig}$ with the leak tube in the down position. These data are presented in Table A-6 (see Appendix A) and Figure 11.

\section{-- Summary of Simulated Crack Experiments}

The experiments conducted during this reporting period complete the current series of simulated crack experiments. Additional experiments may be planned pending a statistical analysis of these data. Although the analysis has been initiated, a detailed discussion of the results and their interpretation is not feasible at this time.

The simulated crack experiments were conducted at initial helium leak rates of $9.8,11.4,11.6,13.2$, and $17.3 \mathrm{cc} / \mathrm{sec}$ at $920 \mathrm{psig}$. The results of experiments at the first three leak rates were presented in the Seventh Quarterly Progress Report (April - June, 1978). The parametric matrix of experimental conditions presented in Table A-7 (see Appendix A) serves as a summary of the completed experiments. A total of 92 experiments have been conducted.

The standard deviation and the mean amount of $\mathrm{PuO}_{2}$ leaked are listed in Table A-8 (see Appendix A) as are the coordinates of these data points. Each set of numbers in the table corresponds to a single set of experimental conditions, under which two or more tests were conducted. Consistent with observations made for the orifice data (Proposal No. 585-3-4069R), the standard deviation for a given experimental condition is directly proportional to the amount of $\mathrm{PuO}_{2}$ leaked, as shown in Figure 12. Further statistical analysis and data interpretation are in progress.

\section{- Future Hork}

The statistical analysis and data interpretation of the simulated crack data will continue. Experiments to complete the four-parameter matrix will continue. 


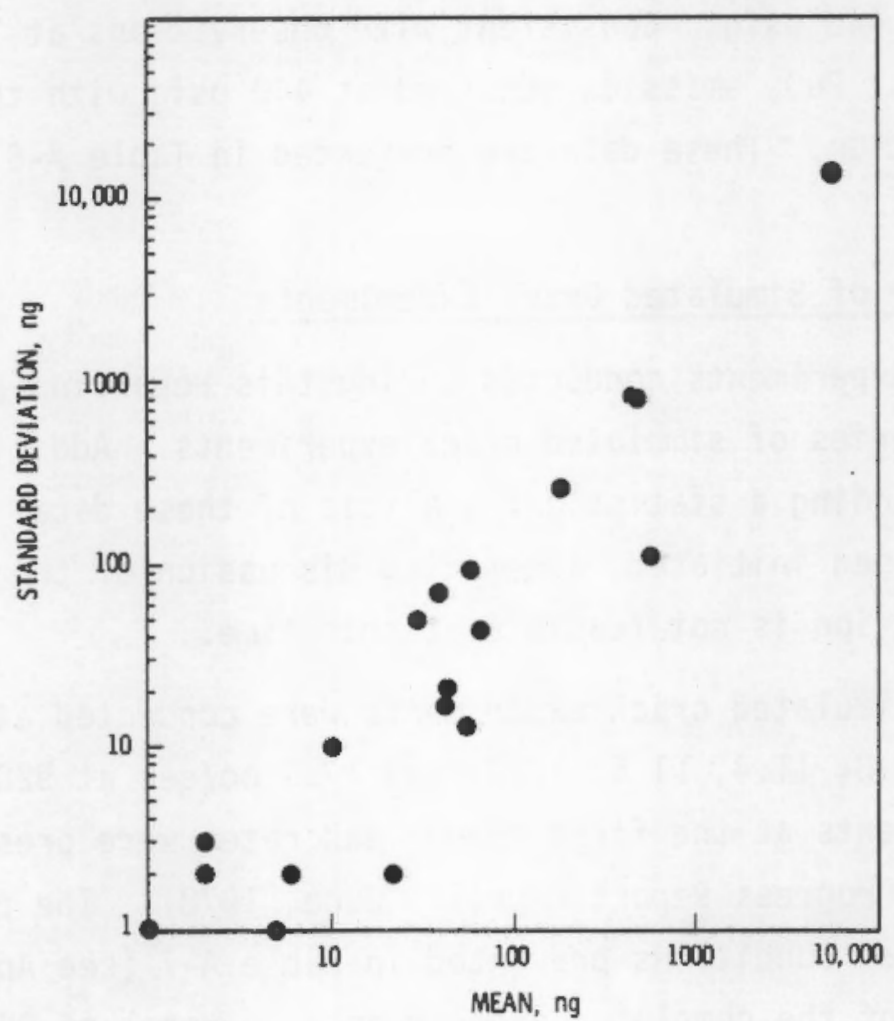

FIGURE 12 -- Standard Deviation Versus Mean For Simulated Crack Experiments 


\section{REFERENCES}

(1) L.C. Schwendiman, et al. Quarterly Progress Report, April 1 through June 30,1978 -- Study of Plutonium Dioxide Leak Rates from Shipping Containers, PNL-2260-7, Battelle, Pacific Northwest Laboratory, Richland, Washington 99352, August 29, 1978.

(2) H.J. Bomelburg. Estimation of Gas Leak Rates Through Very Small Orifices and Channels, BNWL-2223, Battelle, Pacific Northwest Laboratories, Richland, Washington 99352. 
APPENDIX A

TABLES 


\section{TABLE A-1 -- Depleted Uranium Dioxide Transmitted}

Through Well Characterized Leaks

\begin{tabular}{|c|c|c|c|c|c|c|c|c|}
\hline APLA & $\begin{array}{c}\text { ORIFICE } \\
\text { OR CAPILLARY } \\
\text { DESIGNATION } \\
\end{array}$ & $\begin{array}{c}\text { MEASURED } \\
\text { DIAMETER } \\
\mu \mathrm{m} \\
\end{array}$ & $\begin{array}{c}\text { CHAMBER } \\
\text { PRESSURE } \\
\text { psig } \\
\end{array}$ & $\begin{array}{l}\text { TIME } \\
\text { min }\end{array}$ & $\begin{array}{l}\text { AIR FLOW } \\
\text { Cc/min }\end{array}$ & $\begin{array}{l}\text { TRANSMITTED } \\
\text { DUO. } \mu \mathrm{g} \\
\end{array}$ & $\begin{array}{l}\text { Duo } \\
\mu \mathrm{g} / \mathrm{min} \\
\end{array}$ & $\begin{array}{l}\text { DuO } \\
\mu \text { gice }\end{array}$ \\
\hline 1 & $1-110$ & 100 & 1000 & 20 & $7100^{(2)}$ & $5.23+1.6$ & 0.26 & $4 \times 10^{-5}$ \\
\hline 2 & $1-200$ & 200 & 1000 & 20 & $16400^{(2)}$ & $2.5 \times 10^{4} \pm 8.7 \times 10^{3}$ & $1.25 \times 10^{3}$ & $8 \times 10^{-3}$ \\
\hline $3^{(1)}$ & $1-110$ & 100 & 1000 & 10 & $2100^{(2)}$ & $140 \pm 42$ & 14 & $7 \times 10^{-3}$ \\
\hline 4 & $2-110$ & 125 & 500 & 30 & $4300-3800^{(3)}$ & $1330 \pm 410$ & 44 & $1 \times 10^{-2}$ \\
\hline 5 & $2-200$ & 200 & 500 & 30 & $6200^{(2)}$ & $1300 \pm 340$ & 43 & $7 \times 10^{-3}$ \\
\hline 6 & $2-63.5$ & 61 & 1000 & 10 & 2100 & $55.6 \pm 17$ & 6 & $3 \times 10^{-3}$ \\
\hline 7 & & & & . & & & & \\
\hline $7-1$ & $1-20$ & 22 & 500 & 10 & 111 & $60.7 \pm 18$ & 6 & $5 \times 10^{-2}$ \\
\hline $7-2$ & $1-20 a$ & 23 & 500 & 30 & 133 & $16.4 \pm 4.9$ & 0.5 & $3.8 \times 10^{-3}$ \\
\hline $7-3$ & $2-20$ & 20 & 500 & 60 & 94 & 36. $7 \pm 11$ & 0.6 & $6.5 \times 10^{-3}$ \\
\hline $7-4$ & $3-20$ & 23 & 500 & 120 & 140 & $10.6 \pm 3.2$ & 0.1 & $7.1 \times 10^{-4}$ \\
\hline 8 & $2-20$ & 20 & 1000 & 10 & 210 & $13.2 \pm 4$ & 1.3 & $6 \times 10^{-3}$ \\
\hline 9 & $2-36$ & 33 & 1000 & 10 & 580 & $33.1 \pm 9.9$ & 3.3 & $6 \times 10^{-3}$ \\
\hline 10 & $2-63.5$ & 61 & 500 & 30 & 1000 & $54.7 \pm 16$ & 1.8 & $2 \times 10^{-3}$ \\
\hline 11 & $3-36$ & 38 & 500 & 30 & 470 & $21.7 \pm 6.5$ & 0.7 & $2 \times 10^{-3}$ \\
\hline 12 & $2-20$ & 20 & 500 & 30 & 94 & $42.8 \pm 13$ & 1.4 & $2 \times 10^{-2}$ \\
\hline 13 & $1-200$ & 200 & 1000 & 10 & 22000 & $5090 \pm 2600$ & 509 & $2 \times 10^{-2}$ \\
\hline 14 & $250 \mathrm{~A}$ & 274 & 500 & 30 & $5400^{21}$ & $30.4 \pm 9.1$ & 1 & $2 \times 10^{-4}$ \\
\hline 15 & $150 \mathrm{~A}$ & 189 & 1000 & 10 & 10500 & $1280 \pm 660$ & 128 & $1.2 \times 10^{-2}$ \\
\hline 16 & $2-200$ & 200 & 1000 & 10 & 22000 & $8730 \pm 4400$ & 873 & $4 \times 10^{-2}$ \\
\hline 17 & $1-200$ & 200 & 30 & 60 & 1000 & $80.3 \pm 24$ & 1.3 & $1 \times 10^{-3}$ \\
\hline 18 & $1-110$ & 100 & 30 & 60 & 245 & $56.3 \pm 17$ & 0.9 & $4 \times 10^{-3}$ \\
\hline 19 & $1-63.5$ & 66 & 30 & 60 & 80 & $17.8 \pm 5.3$ & 0.3 & $4 \times 10^{-3}$ \\
\hline
\end{tabular}

(1) DUPLICATE OF I BECAUSE OF POOR PRESSURE CONTROL IN RUN 1

(2) LESS THAN DESIRED FLOW

(3) DROPPED I MINUTE INTO RUN

(4) THE \pm IS THE UNCERTAINTY IN THE URANIUM ANALYSIS AT THE 20 CONFIDENCE LEVEL. 
TABLE A-1 (Continued)

\begin{tabular}{|c|c|c|c|c|c|c|c|c|}
\hline APLA & $\begin{array}{l}\text { ORIFICE } \\
\text { OR CAPILLARY } \\
\text { DESIGNATION }\end{array}$ & $\begin{array}{c}\text { MEASURED } \\
\text { DIAMETER } \\
\mu \mathrm{m} \\
\end{array}$ & $\begin{array}{c}\text { CHAMBER } \\
\text { PRESSURE } \\
\text { pSig } \\
\end{array}$ & $\begin{array}{c}\text { TIME } \\
\min \end{array}$ & $\begin{array}{l}\text { AIR FLOW } \\
\text { cclmin }\end{array}$ & $\begin{array}{l}\text { TRANSMITTEO } \\
\text { DUL, } \\
\end{array}$ & $\begin{array}{l}\text { ovo } \\
\mu \mathrm{g} / \mathrm{min}\end{array}$ & $\begin{array}{l}\text { DUO } \\
\mu \mathrm{g} / \mathrm{cc}\end{array}$ \\
\hline \multicolumn{9}{|l|}{20} \\
\hline $20-1$ & $3-30$ & 33 & 500 & 10 & 350 & $19.3 \pm 5.8$ & 1.9 . & $5 \times 10^{-3}$ \\
\hline $20-2$ & $1-36$ & 43 & 500 & 30 & 435 & $32.2 \pm 9.7$ & 1.1 & $3 \times 10^{-3}$ \\
\hline $20-3$ & $2-36$ & 33 & 500 & 60 & 285 & $10.3 \pm 3.1$ & 0.2 & $6 \times 10^{-4}$ \\
\hline $20-4$ & $3-36$ & 38 & 500 & 120 & 470 & $15 \pm 4.5$ & 0.1 & $3 \times 10^{-4}$ \\
\hline 21 & $1-200$ & -200 & 500 & 30 & $6400^{121}$ & $12.8 \pm 3.8$ & 0.4 & $7 \times 10^{-5}$ \\
\hline \multicolumn{9}{|l|}{22} \\
\hline $22-1$ & 3-30 & 33 & 500 & 30 & 350 & $10.5 \pm 3.2$ & 0.5 & $1 \times 10^{-3}$ \\
\hline $22-2$ & $1-36$ & 43 & 500 & 30 & 435 & $20.4 \pm 6.1$ & 0.7 & $2 \times 10^{-3}$ \\
\hline $22-3$ & $2-36$ & 33 & 500 & 30 & 285 & $12.3 \pm 3.7$ & 0.4 & $1 \times 10^{-3}$ \\
\hline $22-4$ & $3-36$ & 38 & 500 & 30 & 470 & $11.3 \pm 3.4$ & 0.4 & $9 \times 10^{-4}$ \\
\hline 23 & $250 \mathrm{~A}$ & 274 & 1000 & 10 & $0-1000^{121}$ & $12.7 \pm 3.8$ & 1.3 & 1. $3 \times 10^{-3}$ \\
\hline \multicolumn{9}{|l|}{24} \\
\hline $24-1$ & $1-20$ & 22 & 500 & 30 & 111 & $7.9 \pm 2.4$ & 0.3 & $2 \times 10^{-3}$ \\
\hline $24-2$ & $2-20$ & 20 & 500 & 30 & 94 & $5.5 \pm 1.6$ & 0.2 & $2 \times 10^{-3}$ \\
\hline $24-3$ & $3-20$ & 23 & 500 & 30 & 140 & $4.5 \pm 1.3$ & 0.2 & $1 \times 10^{-3}$ \\
\hline $24-4$ & $1-20 a$ & 23 & 500 & 30 & 133 & $10.9 \pm 3.3$ & 0.4 & $3 \times 10^{-3}$ \\
\hline \multicolumn{9}{|l|}{25} \\
\hline $25-1$ & $1-63.5$ & 60 & 500 & 30 & 1100 & $75.6 \pm 23$ & 2.5 & $2 \times 10^{-3}$ \\
\hline $25-2$ & $2-63.5$ & 61 & 500 & 30 & 1000 & $78.7 \pm 24$ & 2.6 & $3 \times 10^{-3}$ \\
\hline $25-3$ & $3-63.5$ & 65 & 500 & 30 & 1200 & $87.3 \pm 26$ & 2.9 & $2 \times 10^{-3}$ \\
\hline 26 & $150 B$ & 176 & 500 & 30 & 5600 & $720 \pm 330$ & 24 & $4 \times 10^{-3}$ \\
\hline \multicolumn{9}{|l|}{27} \\
\hline $27-1$ & $1-63.5$ & 66 & 500 & 10 & 1100 & $4 \pm 1.2$ & 0.4 & $4 \times 10^{-4}$ \\
\hline $27-2$ & $2-63.5$ & 61 & 500 & 30 & 1000 & $6.1 \pm 1.8$ & 0.2 & $2 \times 10^{-4}$ \\
\hline $27-3$ & 3-63.5 & 65 & 500 & 60 & 1200 & $10.8 \pm 3.2$ & 0.2 & $2 \times 10^{-4}$ \\
\hline rs & 2008 & 231 & 1000 & 10 & 20000 & $2690 \pm 1100$ & 269 & $1 \times 10^{-2}$ \\
\hline
\end{tabular}


TABLE A-1 (Continued)

\begin{tabular}{|c|c|c|c|c|c|c|c|c|}
\hline APLA & $\begin{array}{l}\text { ORIFICE } \\
\text { OR CAPILLARY } \\
\text { DESIGNATION } \\
\end{array}$ & $\begin{array}{c}\text { MEASURED } \\
\text { DIAMETER } \\
\mu \mathrm{m} \\
\end{array}$ & $\begin{array}{c}\text { CHAMBER } \\
\text { PRESSURE } \\
\text { pSig } \\
\end{array}$ & $\begin{array}{c}\text { TIME } \\
\text { min } \\
\end{array}$ & $\begin{array}{l}\text { AIR FLOW } \\
\text { cc/min }\end{array}$ & $\begin{array}{l}\text { TRANSMITTEO } \\
\text { DUO, }{ }^{(4)} \\
\end{array}$ & $\begin{array}{l}\text { DUO } \\
\mu \mathrm{g} / \mathrm{min}\end{array}$ & $\begin{array}{l}\text { DuO } \\
\mu \mathrm{g} / \mathrm{CC}\end{array}$ \\
\hline \multicolumn{9}{|l|}{29} \\
\hline $29-1$ & $1-20$ & 22 & 1000 & 10 & 275 & $36.5 \pm 11$ & 40 & $1 \times 10^{-2}$ \\
\hline $29-2$ & $1-20 \mathrm{a}$ & 23 & 1000 & 10 & 275 & $9.2 \pm 2.8$ & 0.9 & $3 \times 10^{-3}$ \\
\hline $29-3$ & $2-20$ & 20 & 1000 & 10 & 210 & $20.9 \pm 6.3$ & 2.1 & $1 \times 10^{-2}$ \\
\hline $29-4$ & $3-20$ & 23 & 1000 & 10 & 290 & $6.0 \pm 1.8$ & 0.6 & $2 \times 10^{-3}$ \\
\hline \multicolumn{9}{|l|}{$3 v$} \\
\hline $30-1$ & $1-20$ & 22 & 1000 & 10 & 275 & $6.8 \pm 2$ & 0.7 & $3 \times 10^{-3}$ \\
\hline $30-2$ & $1-20 a$ & 23 & 1000 & 20 & 275 & $3.3 \pm 1.2$ & 0.2 & $7 \times 10^{-4}$ \\
\hline $30-3$ & $2-20$ & 20 & 1000 & 30 & 210 & $13.2 \pm 3.9$ & 0.4 & $2 \times 10^{-3}$ \\
\hline $30-4$ & $3 \cdot 20$ & 23 & 1000 & 60 & 290 & $5.8 \pm 1.7$ & 0.1 & $3 \times 10^{-4}$ \\
\hline \multicolumn{9}{|l|}{31} \\
\hline $31-1$ & $1-20$ & 22 & 30 & 10 & 10 & $7.3 \pm 2.2$ & 0.73 & $7 \times 10^{-2}$ \\
\hline $31-2$ & $1-20 \mathrm{a}$ & 23 & 30 & 30 & 10.3 & $8.5 \pm 2.5$ & 0.28 & $3 \times 10^{-2}$ \\
\hline $31-3$ & $2-20$ & 20 & 30 & 60 & 9.5 & $10.9 \pm 3.3$ & 0.18 & $2 \times 10^{-2}$ \\
\hline $31-4$ & $3-20$ & 23 & 30 & 120 & 14.5 & $9.8 \pm 2.9$ & 0.08 & $6 \times 10^{-3}$ \\
\hline 32 & $1-36$ & 43 & 30 & 60 & 20 & $14.2 \pm 4.3$ & 0.24 & $1 \times 10^{-2}$ \\
\hline \multicolumn{9}{|l|}{33} \\
\hline $33-1$ & $1-20$ & 22 & 30 & 60 & 10 & $<1.6 \pm 1.6$ & $<0.03$ & $<3 \times 10^{-3}$ \\
\hline $33-2$ & $1-200$ & 23 & 30 & 60 & 10.3 & $2.4 \pm 1.6$ & 0.04 & $4 \times 10^{-3}$ \\
\hline $33-3$ & $2-20$ & 20 & 30 & 60 & 9.5 & $<1.6 \pm 1.6$ & $<0.03$ & $<3 \times 10^{-3}$ \\
\hline $33-4$ & $3-20$ & 23 & 30 & 60 & 14.5 & $6.0 \pm 1.8$ & 0.1 & $7 \times 10^{-3}$ \\
\hline 34 & $250 \mathrm{~A}$ & 274 & 30 & 60 & $1180(5)$ & $2.4 \pm 1.6$ & 0.04 & $3 \times 10^{-5}$ \\
\hline \multicolumn{9}{|l|}{35} \\
\hline $35-1$ & $3-30$ & 33 & 30 & 10 & 28 & $3.8 \pm 1.6$ & 0.4 & $1 \times 10^{-2}$ \\
\hline $35-2$ & $1-36$ & 43 & 30 & 30 & 22 & $2.9 \pm 1.6$ & 0.1 & $5 \times 10^{-3}$ \\
\hline
\end{tabular}

(5) Through flow was achieved wire could not be run through, appears plugged after run 
TABLE A-1 (Continued)

\begin{tabular}{|c|c|c|c|c|c|c|c|c|c|}
\hline \multirow{2}{*}{\multicolumn{2}{|c|}{ 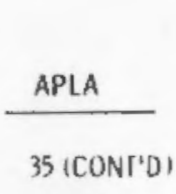 }} & \multirow[t]{2}{*}{$\begin{array}{l}\text { ORIFICE } \\
\text { OR CAPILLARY } \\
\text { DESIGNAIION }\end{array}$} & \multirow[t]{2}{*}{$\begin{array}{l}\text { MEASURED } \\
\text { DIAMETER } \\
\mu \mathrm{m}\end{array}$} & \multirow[t]{2}{*}{$\begin{array}{c}\text { CHAMBER } \\
\text { PRESSURE } \\
\text { psig }\end{array}$} & \multirow[t]{2}{*}{$\begin{array}{c}\text { IIME } \\
\text { min }\end{array}$} & \multirow[t]{2}{*}{$\begin{array}{l}\text { AIR FLOW } \\
\mathrm{cc} / \mathrm{min}\end{array}$} & \multirow[t]{2}{*}{$\begin{array}{l}\text { TRANSMITTED } \\
\text { DUO, } \\
\text { (4) }\end{array}$} & \multirow[t]{2}{*}{$\begin{array}{l}\text { DUO } \\
\mu g / \min \end{array}$} & \multirow[t]{2}{*}{$\begin{array}{l}\text { DUO } \\
\mu \mathrm{g} / \mathrm{Cc}\end{array}$} \\
\hline & & & & & & & & & \\
\hline & $35-3$ & $2-36$ & 33 & 30 & 60 & 24 & $2.6 \pm 1.6$ & 0.04 & $2 \times 10^{-3}$ \\
\hline & $35-4$ & $3-36$ & 38 & 30 & 120 & 39 & $20.1 \pm 6$ & 0.2 & $5 \times 10^{-3}$ \\
\hline 36 & & $1-20$ & 22 & 30 & 60 & 10 & $4.1 \pm 1.6$ & 0.07 & $7 \times 10^{-3}$ \\
\hline 37 & & $250 \mathrm{~B}$ & 274 & 30 & 60 & 520 & $5.9 \pm 1.8$ & 0.1 & $2 \times 10^{-4}$ \\
\hline 38 & $38-1$ & $3-30$ & 33 & 30 & 60 & 28 & $3.1 \pm 1.6$ & 0.05 & $2 \times 10^{-3}$ \\
\hline & $38-2$ & $1-36$ & 43 & 30 & 60 & 22 & $2.0 \pm 1.6$ & 0.03 & $1 \times 10^{-3}$ \\
\hline & $38-3$ & $2-36$ & 33 & 30 & 60 & 24 & $17.5 \pm 5.3$ & 0.3 & $1 \times 10^{-2}$ \\
\hline & $38-4$ & $3-36$ & 38 & 30 & 60 & 39 & $43.7 \pm 1.3$ & 0.7 & $2 \times 10^{-2}$ \\
\hline \multicolumn{10}{|c|}{39} \\
\hline & 341 & 1.63 .5 & 66 & $1000 \bullet 0$ & 1200 & -. & $303 \pm 91$ & 0.3 & .. \\
\hline & $39-2$ & $2-63.5$ & 6) & $1000 \bullet 0$ & 1200 & $\cdot-$ & $49.8 \pm .5$ & 0.04 & -. \\
\hline & $39-3$ & 3.63 .5 & 65 & $1000 \rightarrow 0$ & 1200 & $\cdots$ & $326 \pm 98$ & 0.3 & -. \\
\hline \multicolumn{10}{|c|}{40} \\
\hline & $40-1$ & $1-63.5$ & 66 & 1000 & 10 & 2300 & $43.2 \pm 13$ & 4.3 & $2 \times 10^{-3}$ \\
\hline & $40-2$ & $2-63.5$ & 61 & 1000 & 10 & 860121 & $20.3 \pm 6.1$ & 2.0 & $2 \times 10^{-3}$ \\
\hline & $40-3$ & $3-63.5$ & 65 & 1000 & 10 & $176012 !$ & $76.8 \pm 23$ & 1.7 & $4 \times 10^{-3}$ \\
\hline 41 & & $150 \mathrm{~s}$ & 182 & 1000 & 10 & 14600 & $212 \pm 64$ & 21.2 & $1 \times 10^{-3}$ \\
\hline \multicolumn{10}{|c|}{42} \\
\hline & $42-1$ & $1-63.5$ & 66 & 1000 & 10 & $200(2)$ & $17.8 \pm 5.3$ & 2 & $1 \times 10^{-2}$ \\
\hline & $42-2$ & $2-63.5$ & 61 & 1000 & 30 & $1450(2)$ & $61.9 \pm 19$ & 2 & $1 \times 10^{-3}$ \\
\hline & $42-3$ & $3-63.5$ & 65 & 1000 & 60 & 2400 & $167 \pm 50$ & 3 & $1 \times 10^{-3}$ \\
\hline 43 & & 1505 & 182 & 500 & 30 & 5600 & $290 \pm 87$ & 9.7 & $2 \times 10^{-3}$ \\
\hline 44 & & $150 B$ & 176 & 30 & 60 & 520 & $12.4 \pm 3.7$ & 0.2 & $4 \times 15^{-4}$ \\
\hline
\end{tabular}


TABLE A-1 (Continued)

\begin{tabular}{|c|c|c|c|c|c|c|c|c|}
\hline APLA & $\begin{array}{l}\text { ORIFICE } \\
\text { OR CAPILIARY } \\
\text { DESIGNATION }\end{array}$ & $\begin{array}{c}\text { MEASURED } \\
\text { DIAMETER } \\
\mu \mathrm{m} \\
\end{array}$ & $\begin{array}{c}\text { CHAMBER } \\
\text { PRESSURE } \\
\text { psig } \\
\end{array}$ & $\begin{array}{l}\text { TIME } \\
\text { min }\end{array}$ & $\begin{array}{l}\text { AIR FLOW } \\
\text { ccimin }\end{array}$ & $\begin{array}{l}\text { TRANSMITIEO (4) } \\
\text { DU0. } \mu 9\end{array}$ & $\begin{array}{l}\text { ouo } \\
\mu g / \text { min }\end{array}$ & $\begin{array}{l}\text { Dul } \\
\mu g / C c\end{array}$ \\
\hline 45 & $150 \mathrm{~S}$ & 182 & 30 & 60 & 640 & $18.0 \pm 5.4$ & 0.3 & $5 \times 10^{-4}$ \\
\hline \multicolumn{9}{|l|}{46} \\
\hline $46-1$ & $3-30$ & 33 & 1000 & 10 & 695 & $11.5+3.5$ & 1.2 & $2 \times 10^{-3}$ \\
\hline $46-2$ & $1-36$ & 43 & 1000 & 20 & 840 & $97.3 \pm 29$ & 4.9 & $6 \times 10^{-3}$ \\
\hline $46-3$ & $2-36$ & 33 & 1000 & 30 & 580 & $10.7 \pm 3.2$ & 0.4 & $6 \times 10^{-4}$ \\
\hline $46-4$ & $3-36$ & 38 & 1000 & 60 & 920 & $32.6+9.8$ & 0.5 & $6 \times 10^{-4}$ \\
\hline \multicolumn{9}{|l|}{47} \\
\hline $47-1$ & $3-30$ & 33 & 1000 & 10 & 695 & $33.6 \pm 10$ & 3.4 & $5 \times 10^{-3}$ \\
\hline $47-2$ & $1-36$ & 43 & 1000 & 10 & 840 & $20.5 \pm 0.2$ & 2.0 & $2 \times 10^{-3}$ \\
\hline $47-3$ & $2 \cdot 36$ & 33 & 1000 & 10 & 580 & $1.35 \times 10^{3} \pm 510^{161}$ & & \\
\hline $47-4$ & $3-36$ & 3. & 1000 & 10 & 920 & $0.3 \cdot 1.9$ & 0.0 & $7 \times 10^{-4}$ \\
\hline \multicolumn{9}{|l|}{48} \\
\hline $48-1$ & $1-63,5$ & $\infty 6$ & 30 & 30 & so & b. $19+1.9$ & 0.2 & $3 \times 10^{-3}$ \\
\hline $48-2$ & $2-63.5$ & bl & 30 & 60 & 88 & $15.3 \pm 4.6$ & 0.3 & $3 \times 10^{-4}$ \\
\hline $48-3$ & $3-n 3.5$ & BS & 30 & 120 & 109 & $11.0 \div 3.3$ & 0.09 & $9 \times 10^{-4}$ \\
\hline \multicolumn{9}{|l|}{49} \\
\hline $44-1$ & $1-63.5$ & 66 & 30 & 57 & 80 & $12.2 \pm 3.7$ & 0.2 & $3 \times 10^{-3}$ \\
\hline $49-2$ & 2.63 .5 & 61 & 30 & 57 & 88,71 & $4.3+1.7$ & 0.08 & $9 \times 10^{-4}$ \\
\hline $49-3$ & 3-63.5 & 65 & 30 & 57 & 109 & $25+7.5$ & 0.4 & $4 \times 10^{-3}$ \\
\hline 50 & $100 B$ & 99 & 1000 & 10 & 3140 & $87.8+\not 0$ & 8.8 & $3 \times 10^{-3}$ \\
\hline 51 & & & & & & & & \\
\hline $51-1$ & $1-110$ & 100 & 1000 & 15 & 7100 & $259 \pm 78$ & 17.3 & $2 \times 10^{-3}$ \\
\hline $51-2$ & $2-110$ & 125 & 1000 & 30 & $500 \div 21$ & $401 \pm 120$ & 13.4 & $2 \times 10^{-3}$ \\
\hline $51-3$ & $3-110$ & 100 & 1000 & 40 & 5800 & $250+75$ & 6.3 & $1 \times 10^{-3}$ \\
\hline
\end{tabular}

16) This or ifice inadequately sealed, leaked duo (results omitted!

17) Lost flow after 30 minutes 
TABLE A-1 (Continued)

\begin{tabular}{|c|c|c|c|c|c|c|c|c|c|}
\hline \multicolumn{2}{|c|}{ APLA } & $\begin{array}{c}\text { ORIFICE } \\
\text { OR CAPILLARY } \\
\text { DESIGNATION }\end{array}$ & $\begin{array}{l}\text { MEASURED } \\
\text { DIAMETER } \\
\mu \mathrm{m}\end{array}$ & $\begin{array}{l}\text { CHAMBER } \\
\text { PRESSURE } \\
\text { psig }\end{array}$ & $\begin{array}{l}\text { TIME } \\
\text { min }\end{array}$ & $\begin{array}{l}\text { AIR FLOW } \\
\text { ccímin }\end{array}$ & $\begin{array}{l}\text { TRANSMITEO (4) } \\
\text { DUO. } \mu_{Y}\end{array}$ & $\begin{array}{l}\text { ouo } \\
\mu \mathrm{g} / \mathrm{min}\end{array}$ & $\begin{array}{l}\text { Duo } \\
\mu g^{\prime}{ }^{\prime C C}\end{array}$ \\
\hline \multicolumn{2}{|l|}{52} & $100 \mathrm{~B}$ & 99 & 1000 & 10 & $400+81$ & $6.2+1.9$ & 0.6 & $2 \times 10^{-3}$ \\
\hline \multicolumn{10}{|l|}{53} \\
\hline & $3-1$ & 1.110 & 100 & 1000 & 10 & 7100 & $350+110$ & 35 & $5 \times 10^{-3}$ \\
\hline & $3-2$ & $2-110$ & 125 & 1000 & 10 & 2200121 & $211+63$ & 21.1 & $1 \times 10^{-2}$ \\
\hline & $3-3$ & 3. 110 & 100 & 1000 & 10 & 5800 & $187+56$ & 187 & $3 \times 10^{-3}$ \\
\hline 54 & & $100 \mathrm{~B}$ & 99 & 500 & 30 & 145,21 & $4.07+1.7$ & 0.1 & $7 \times 10^{-4}$ \\
\hline 55 & & $250 \mathrm{~S}$ & $27 \mathrm{n}$ & 1000 & 10 & 38800 & 4. $21 \times 10^{3}+360$ & 921 & $2 \times 10^{-2}$ \\
\hline \multicolumn{10}{|l|}{$t^{k}$} \\
\hline & $x-1$ & $1-200$ & 200 & 1000 & 11 & 33.000 .9$. & & & \\
\hline & $8-2$ & 2200 & 200 & $H(100)$ & 10 & ¿2.000 & $2120 \div \times 20$ & 212 & $1 \times 10^{-2}$ \\
\hline & $8-3$ & 3-200 & 200 & 1000 & 10 & 22000 & $2350 \cdot 900$ & 235 & $1 \times 10^{-2}$ \\
\hline 69 & & $200 \mathrm{~s}$ & $22 \pi$ & 500 & 30 & 8000 & $3340 \div 130$ & 111 & $1 \times 10^{-2}$ \\
\hline \multicolumn{10}{|l|}{72} \\
\hline & $2-1$ & $1-200$ & 200 & 500 & 10 & 12200 & $2380 \pm 910$ & 238 & $2 \times 10^{-2}$ \\
\hline & $2-2$ & $2-200$ & $310 \div 101$ & 500 & 30 & 11200 & $1 \% 0 \pm 760$ & 65 & $6 \times 10^{-3}$ \\
\hline & $2-3$ & $3-200$ & 200 & 500 & bo & 11000 & & & \\
\hline \multicolumn{10}{|l|}{74} \\
\hline & $4-1$ & $1-200$ & 200 & 500 & 30 & 12200 & $3480 \pm 1300$ & 116 & $1 \times 10^{-2}$ \\
\hline & $4-2$ & $2-200$ & $310 \div 111$ & 500 & 30 & 11200 & $2420 \pm 930$ & 81 & $7 \times 10^{-3}$ \\
\hline & $4-3$ & $3-200$ & 200 & 500 & 30 & 11000 & & & \\
\hline \multicolumn{10}{|l|}{76} \\
\hline & $6-1$ & $1-200$ & 200 & 1000 & 10 & 4000121 & $1690 \pm 660$ & 169 & $4 \times 10^{-2}$ \\
\hline & $6-2$ & $2-200$ & $310+111$ & 1000 & 30 & 22000 & $4.31 \times 10^{4} \pm 1.6 \times 10^{4}$ & 1436 & $7 \times 10^{-2}$ \\
\hline & $6-3$ & $3-200$ & 200 & 1000 & 60 & $4000-7000(2)$ & $7460 \pm 2800$ & 124 & $2 \times 10^{-2}$ \\
\hline (8) 11 & ncorr & ow & (10) Plugge & un: entar & $10_{\mu m} \mathrm{sq}$ & uring cleaning & & & \\
\hline (9) $\mathrm{P}$ & lugget & Imediately & (11) $310 \mu \pi$ & not correct. & not use & & & & \\
\hline
\end{tabular}


TALLE A-2 -- Depleted Uranium Dioxide Transmission Rates for Leak Paths Uncier the Powder Level

\begin{tabular}{|c|c|c|c|c|c|c|c|c|c|}
\hline UPL & $\begin{array}{c}\text { ORIFICE } \\
\text { OR CAPILLARY } \\
\text { DESIGNAIION } \\
\end{array}$ & $\begin{array}{c}\text { MEASURED } \\
\text { DIAMETER, } \\
\mu \mathrm{m} \\
\end{array}$ & $\begin{array}{c}\text { CHAMBER } \\
\text { PRESSURE } \\
\text { psig } \\
\end{array}$ & AGITAIION & $\begin{array}{l}\text { LENGTH } \\
\text { OF RUN } \\
\text { min }\end{array}$ & $\begin{array}{c}\text { AIR FLOW } \\
\text { ccimin }\end{array}$ & $\begin{array}{l}\text { IRANSMITTED(3) } \\
\text { DUO, } \mu \mathrm{g}\end{array}$ & $\begin{array}{l}\text { DUO } \\
\mu \mathrm{g} / \min \\
\end{array}$ & $\begin{array}{l}\text { DUO } \\
\mu \mathrm{g} / \mathrm{CC} \\
\end{array}$ \\
\hline 1 & $2-200$ & 200 & 1000 & NO & 5 & $12200-21000$ & $3.54 \times 10^{3} \pm 1.3 \times 10^{3}$ & (1) & (1) \\
\hline 2 & $2-200$ & 200 & 1000 & NO & 5 & 13800 & $1.61 \times 10^{3} \pm 5.9 \times 10^{2}$ & $3.2 \times 10^{2}$ & $2 \times 10^{-2}$ \\
\hline 3 & $2-200$ & 200 & 1000 & YES & 5 & 6200 & $4.89 \times 10^{3} \pm 1.7 \times 10^{3}$ & $9.8 \times 10^{2}$ & $2 \times 10^{-1}$ \\
\hline 4 & $2-200$ & 200 & 5 & NO & 60 & 16.6 & $6 . \% \pm 2.1$ & 0.1 & $7 \times 10^{-3}$ \\
\hline 5 & $2-200$ & 200 & 5 & YES & 60 & 18.2 & $4.05 \pm 1.2$ & 0.1 & $4 \times 10^{-3}$ \\
\hline 6 & $2-110$ & 125 & 1000 & NO & 20 & 4600 & $118 \pm 35$ & 5.9 & $1 \times 10^{-3}$ \\
\hline 7 & $2-110$ & 125 & 1000 & YES & 20 & 8000 & $133 \pm 40$ & 6.7 & $8 \times 10^{-4}$ \\
\hline 8 & $2-110$ & 125 & 15 & YES & 60 & 76 & $37.2 \pm 11$ & 0.6 & $8 \times 10^{-3}$ \\
\hline 9 & $2-110$ & 125 & 15 & NO & 60 & 18 & $25.2 \pm 7.5$ & 0.4 & $2 \times 10^{-2}$ \\
\hline 10 & $2-63.5$ & 61 & 1000 & NO & 20 & 1900 & $58.9 \pm 18$ & 2.9 & $2 \times 10^{-3}$ \\
\hline 11 & $2-63.5$ & 61 & 1000 & YES & 20 & 2000 & $111 \pm 33$ & 5.6 & $3 \times 10^{-3}$ \\
\hline 12 & $2-63.5$ & 61 & 15 & NO & 60 & 18 & $51.5 \pm 1.5$ & 1.0 & $5 \times 10^{-2}$ \\
\hline 13 & $2-63.5$ & 61 & 15 & YES & 60 & 16 & $17.4 \pm 5$ & 0.3 & $2 \times 10^{-2}$ \\
\hline 14 & $2-36$ & 33 & 1000 & NO & 20 & 31 & $14.8 \pm 4$ & 0.7 & $2 \times 10^{-2}$ \\
\hline 15 & $2 \cdot 36$ & 33 & 1000 & YES & 20 & 26 & $72.5 \pm 22$ & 3.6 & $1 \times 10^{-2}$ \\
\hline 16 & $2-36$ & 33 & 15 & NO & 120 & 0.3 & $4.5 \pm 1.8$ & 0.04 & $1 \times 10^{-1}$ \\
\hline 17 & $2-36$ & 33 & 15 & YES & 120 & 1.1 & $12.6 \pm 3.8$ & 0.1 & $9 \times 10^{-2}$ \\
\hline 18 & $2-20$ & 20 & 1000 & NO & 20 & 30 & $82.4 \pm 25$ & 4.0 & $1 \times 10^{-1}$ \\
\hline 19 & $2-20$ & 20 & 1000 & YES & 20 & 61 & $11.2 \pm 3.4$ & 0.6 & $1 \times 10^{-2}$ \\
\hline 20 & $2-20$ & 20 & 15 & YES & 120 & No ${ }^{(2)}$ & $21.2 \pm 6$ & 0.2 & \\
\hline 21 & $2-20$ & 20 & 15 & NO & 120 & ND & $13.6 \pm 4$ & 0.1 & \\
\hline 22 & $2-200$ & 200 & 500 & NO & 20 & 6500 & $812 \pm 220$ & 40.6 & $6 \times 10^{-3}$ \\
\hline 23 & $2-200$ & 200 & 500 & YES & 20 & 5750 & $1 \% 0 \pm 330$ & 98.0 & $2 \times 10^{-2}$ \\
\hline 24 & $2-110$ & 185 & 500 & NO & 20 & 3250 & $150 \pm 45$ & 7.5 & $2 \times 10^{-3}$ \\
\hline
\end{tabular}

(1) ALMOST ALL DUO EXPELLED IMMEDIATELY

(2) ND, NOT DETECTABLE, LESS IHAN 0.2 ccimin

(3) THE \pm IS THE UNCERTAINTY IN THE URANIUM ANALYSIS AT THE $2 \sigma$ CONFIDENCE LEVEL 
TABLE A-2 (Continued)

\begin{tabular}{|c|c|c|c|c|c|c|c|c|c|}
\hline UPL & $\begin{array}{c}\text { ORIFICE } \\
\text { OR CAPILLARY } \\
\text { DESIGNAIION } \\
\end{array}$ & $\begin{array}{l}\text { MEASURED } \\
\text { DIAMETER, } \\
\mu \mathrm{m}\end{array}$ & $\begin{array}{c}\text { CHAMBER } \\
\text { PRESSURE } \\
\quad \text { psig } \\
\end{array}$ & AGITAIION & $\begin{array}{l}\text { LENGIH } \\
\text { OF RUN, } \\
\text { min } \\
\end{array}$ & $\begin{array}{l}\text { AIR FLOW } \\
\mathrm{cc} / \mathrm{min}\end{array}$ & $\begin{array}{l}\text { TRANSMITED (3) } \\
\text { DUO, } \mu \mathrm{g} \\
\end{array}$ & $\begin{array}{l}\text { DU0 } \\
\mu g / \min \end{array}$ & $\begin{array}{l}\text { Duo } \\
\mu \mathrm{gicc} \\
\end{array}$ \\
\hline 25 & $2-110$ & 125 & 500 & YES & 20 & 2550 & $86.3 \pm 26$ & 4.3 & $2 \times 10^{-3}$ \\
\hline 26 & $2-63.5$ & 61 & 500 & NO & 30 & 470 & $71.8 \pm 22$ & 2.4 & $5 \times 10^{-3}$ \\
\hline 27 & $2-63.5$ & 61 & 500 & YES & 30 & 275 & $37.0 \pm 11$ & 1.2 & $4 \times 10^{-3}$ \\
\hline 28 & $2-36$ & 33 & 500 & No & 60 & 225 & $20.4 \pm 6$ & 0.3 & $1 \times 10^{-3}$ \\
\hline 29 & $2-36$ & 33 & 500 & YES & 60 & 75 & $36.1 \pm 11$ & 0.6 & $8 \times 10^{-3}$ \\
\hline 30 & $2-20$ & 20 & 500 & NO & 60 & 14 & $15.0 \pm 5$ & 0.3 & $6 \times 10^{-3}$ \\
\hline 31 & $2-20$ & 20 & 500 & YES & 60 & 0.75 & $13.0 \pm 4$ & 0.2 & $3 \times 10^{-1}$ \\
\hline 32 & $250 \mathrm{~B}$ & 274 & 1000 & No & 5 & 32500 & $379 \pm 110$ & 75.8 & $2 \times 10^{-3}$ \\
\hline 33 & $250 \mathrm{~B}$ & 274 & 1000 & YES & 10 & 32000 & $2830 \pm 400$ & 283 & $9 \times 10^{-3}$ \\
\hline 34 & $150 \mathrm{~B}$ & 176 & 1000 & NO & 5 & 9600 & $1270 \pm 220$ & 254 & $3 \times 10^{-2}$ \\
\hline 35 & $150 \mathrm{~B}$ & 176 & 1000 & YES & 10 & 7400 & $498 \pm 120$ & 50 & $7 \times 10^{-3}$ \\
\hline 36 & $250 \mathrm{~B}$ & 274 & 500 & NO & 30 & 14100 & $311 \pm 98$ & 10 & $7 \times 10^{-4}$ \\
\hline 37 & $250 \mathrm{~B}$ & 274 & 500 & YES & 30 & 11500 & $585 \pm 140$ & 20 & $2 \times 10^{-3}$ \\
\hline 38 & $150 \mathrm{~B}$ & 176 & 500 & NO & 30 & 3950 & $374 \pm 100$ & 12 & $3 \times 10^{-3}$ \\
\hline 39 & $150 \mathrm{~B}$ & 176 & 500 & YES & 30 & 5400 & $395 \pm 120$ & 13 & $2 \times 10^{-3}$ \\
\hline 40 & $250 \mathrm{~B}$ & 274 & 15 & YES & 60 & 410 & $1950 \pm 300$ & 33 & $8 \times 10^{-2}$ \\
\hline 41 & $250 \mathrm{~B}$ & 274 & 15 & NO & 60 & 28 & $78.3 \pm 23$ & 1.3 & $4 \times 10^{-2}$ \\
\hline 42 & $150 \mathrm{~B}$ & 176 & 15 & YES & 60 & 29 & $122 \pm 37$ & 2 & $7 \times 10^{-2}$ \\
\hline 43 & $150 \mathrm{~B}$ & 176 & 15 & NO & 60 & 50 & $129 \pm 39$ & 2 & $4 \times 10^{-2}$ \\
\hline 44 & $2-200$ & 200 & 100 & NO & 30 & 820 & $740 \pm 400$ & 25 & $3 \times 10^{-2}$ \\
\hline 45 & $2-200$ & 200 & 100 & YES & 30 & 485 & $578 \pm 320$ & 19 & $3 \times 10^{-2}$ \\
\hline 46 & $2-110$ & 125 & 100 & NO & 30 & 325 & $464 \pm 140$ & 15 & $5 \times 10^{-2}$ \\
\hline 47 & $2-110$ & 125 & 100 & YES & 30 & 280 & $120 \pm 36$ & 4 & $1 \times 10^{-2}$ \\
\hline 48 & $2-63.5$ & 61 & 100 & No & 30 & 26.6 & $68.4 \pm 21$ & 2.3 & $9 \times 10^{-2}$ \\
\hline
\end{tabular}


TABLE A-2 (Continued)

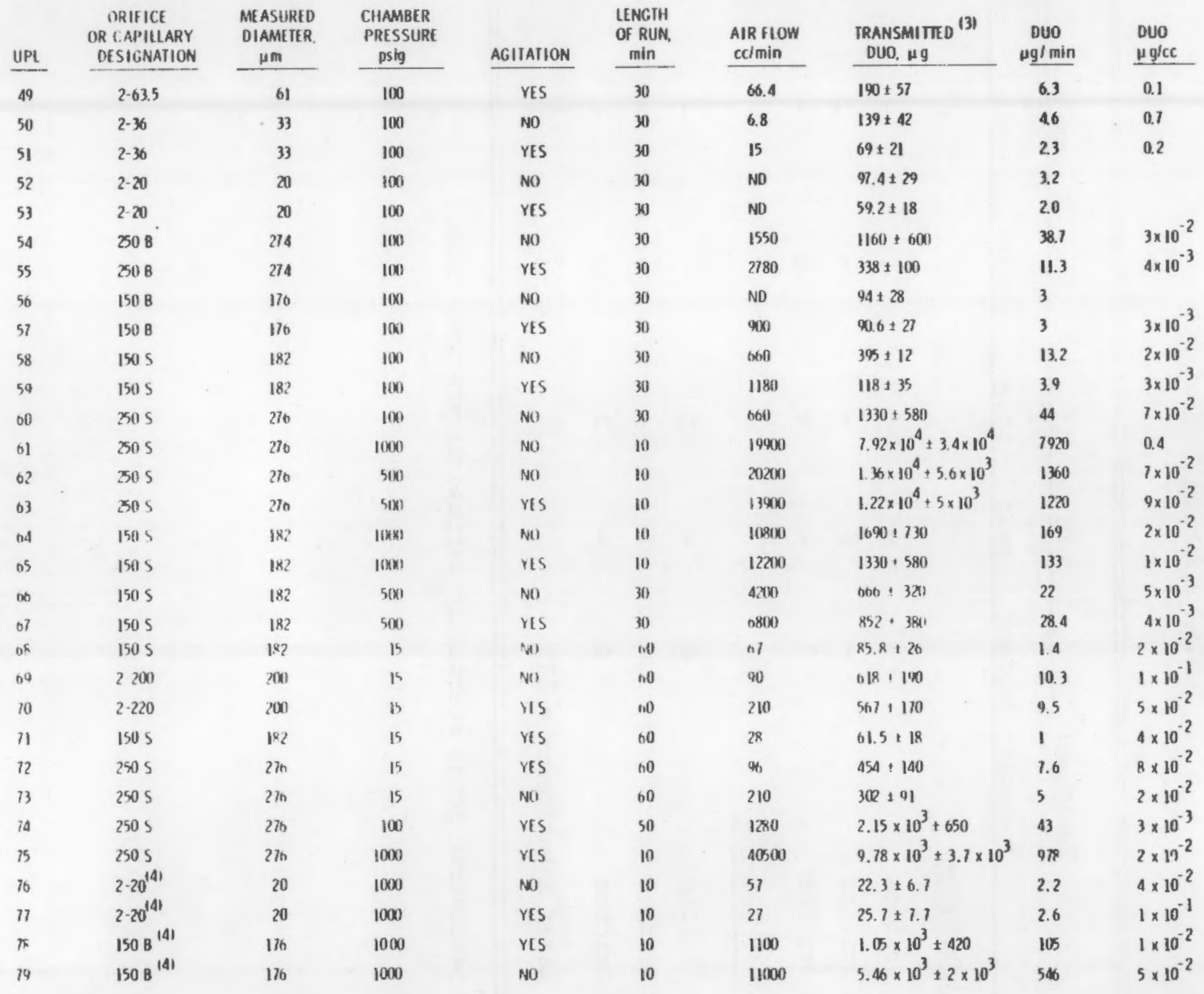


TABLE A-3 -- ADDITIONAI TESTS PROPOSED TO ADEQDATEII DEFTNE PARAMEIRIC RELATIONS

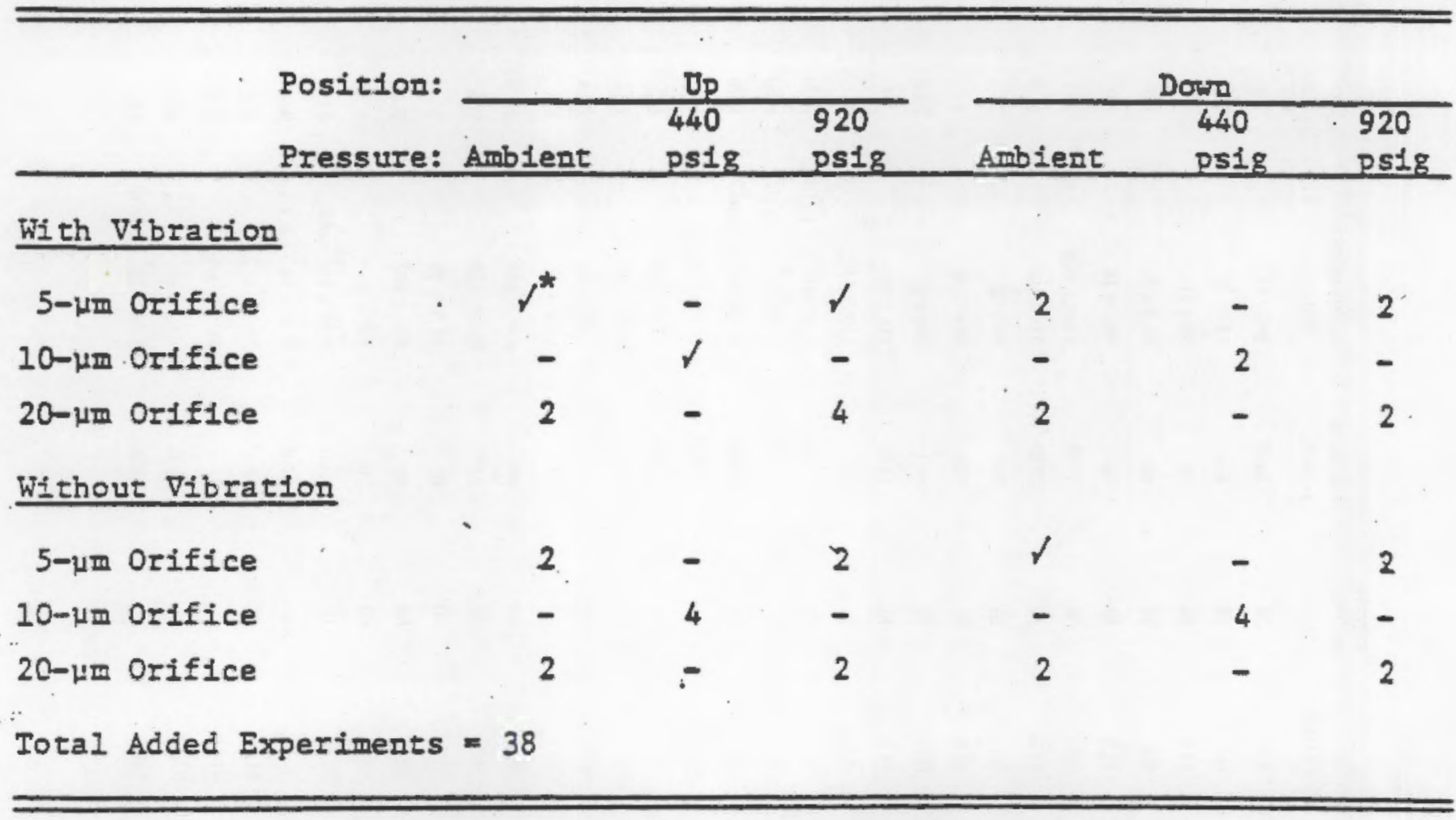

* $\checkmark$ parametric polnt adequately defined by previous tests. 
TABLE A-4 SUMMARY OF $\mathrm{PuO}_{2}$ LEAK RATE EXPERIMENTS USING A 5- $\mu \mathrm{m}$ ORIFICE

\begin{tabular}{|c|c|c|c|c|c|c|c|c|}
\hline \multirow[b]{2}{*}{$\begin{array}{l}\text { Run } \\
\text { Number }\end{array}$} & \multirow[b]{2}{*}{$\begin{array}{l}\text { Tube } \\
\text { Position }\end{array}$} & \multirow[b]{2}{*}{$\begin{array}{l}\text { Helium } \\
\text { Pressure, } \\
\text { psig }\end{array}$} & \multirow[b]{2}{*}{ Vibration } & \multirow{2}{*}{$\begin{array}{l}\text { Helfutn } \\
\text { Leak } \\
\text { Rate (b), } \\
\text { cc/sec }\end{array}$} & \multicolumn{4}{|c|}{ Quantity of $\mathrm{PuO}_{2}$ Detected, $\mathrm{\mu g}_{\mathrm{g}}$ (a) } \\
\hline & & & & & $\begin{array}{l}\text { Inlet } \\
\text { Nozzle }\end{array}$ & $\begin{array}{l}\text { Final } \\
\text { Filter }\end{array}$ & Total & $\stackrel{\text { Net }}{\text { Total (c) }}$ \\
\hline B56 (d) & Sideways & - & - & - & 0.00060 & 0.00079 & 0.00139 & -- \\
\hline$B 57(d)$ & S1deways & - & - & - & 0.00073 & 0.00035 & 0.00108 & 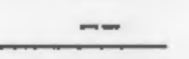 \\
\hline Pu 82 & Up & 0 & No & - & 0.00111 & 0.00313 & 0.00424 & 0.00301 \\
\hline Pu82a & $U_{P}$ & 0 & No & - & 0.00129 & 0.00465 & 0.00594 & 0.00471 \\
\hline Pu83 & Down & 0 & Yes & - & 0.00236 & 0.00712 & 0.00948 & 0.00825 \\
\hline Pu83a & Down & 0 & Yes & - & 0.00662 & 0.01014 & 0.01676 & 0.01553 \\
\hline Pu83b & Down & 0 & Yes & - & 0.00782 & 0.09217 & 0.09999 & 0.09876 \\
\hline Pu83c & Down & 0 & Yes & - & 0.00588 & 0.00054 & 0.00643 & 0.00520 \\
\hline Pu84 & Up & 920 & No & 0.4 & 0.00022 & 0.00069 & 0.00091 & 0.0 \\
\hline Pu84a & Up & 920 & No & 0.4 & 0.00048 & 0.00030 & 0.00078 & 0.0 \\
\hline Pu85 & Down & 920 & Yes & 0.4 & 0.00099 & 0.00180 & 0.00279 & 0.00156 \\
\hline Pu85a & Down & 920 & Yes & 1.7 & 0.00260 & 0.00155 & 0.00415 & 0.00292 \\
\hline Pu86 & Down & 920 & No & 3.9 & 0.00090 & 0.01253 & 0.01344 & 0.01221 \\
\hline Pu86a & Down & 920 & No & 0.9 & 0.00045 & 0.00155 & 0.00200 & 0.00077 \\
\hline Pu86b & Down & 920 & No & 0.4 & 0.00182 & 0.00057 & 0.00239 & 0.00116 \\
\hline Pu86c & Down & 920 & No & 0.4 & 0.00045 & 0.0 & 0.00045 & 0.0 \\
\hline
\end{tabular}

(a) Based on a specific activity of $0.096 \mathrm{CI} / \mathrm{g}$ for the $\mathrm{PuO}_{2}$ powder.

(b) Hellum leak rate determined by pressure decay method at the midpoint of the run.

(c) The net total is the amount ahove the average containment box background of $0.00124 \mu \mathrm{g}$.

(d) Containment box background. 
TABLE A-5 SUMMARY OF ROOM-TEMPERATURE PuO 2 LEAK RATE EXPERIMENTS USING A SIMULATED CRACK (a)

\begin{tabular}{|c|c|c|c|c|c|c|c|c|}
\hline \multirow[b]{2}{*}{$\begin{array}{c}\text { Run } \\
\text { Number }\end{array}$} & \multirow[b]{2}{*}{$\begin{array}{l}\text { Tube } \\
\text { Position }\end{array}$} & \multirow{2}{*}{$\begin{array}{c}\text { Helium } \\
\text { Pressure, } \\
\text { psig }\end{array}$} & \multirow[b]{2}{*}{ Vibration } & \multirow{2}{*}{$\begin{array}{l}\text { Hellum } \\
\text { Leak } \\
\text { Rate, } \\
\text { cc/sec }\end{array}$} & \multicolumn{4}{|c|}{ Quantity of $\mathrm{PuO}_{2}$ Detected, $\mathrm{\mu g}^{(\mathrm{b})}$} \\
\hline & & & & & $\begin{array}{l}\text { Inlet } \\
\text { Nozzle }\end{array}$ & $\begin{array}{l}\text { Final } \\
\text { Filter }\end{array}$ & Total & Total $_{\text {Tot }}$ \\
\hline$B-52(e)$ & Sideways & - & - & - & 0.00035 & 0.00031 & 0.00066 & - \\
\hline$B-53(e)$ & Sideways & - & - & - & 0.00028 & 0 & 0.00028 & - \\
\hline Pu 68 & Up & 920 & No & 12.4 & 0.01082 & 0.09195 & 0.10278 & 0.10231 \\
\hline $\mathrm{Pu} 68 \mathrm{a}$ & Up & 920 & No & 11.3 & 0.00004 & 0.00632 & 0.00636 & 0.00589 \\
\hline $\mathrm{Pu}$ 68b & Up & 920 & No & 9.6 & 0.00120 & 0.00256 & 0.00376 & 0.00329 \\
\hline $\mathrm{Pu} \mathrm{68c}$ & Up & 920 & No & 10.0 & 0 & 0.00333 & 0.00333 & 0.00286 \\
\hline Pu 69 & Up & 920 & Yes & 11.1 & 0.00031 & 0.00795 & 0.00826 & 0.00779 \\
\hline $\mathrm{Pu}$ 69a & Up & 920 & Yes & 9.3 & 0.00109 & 0.00392 & 0.00501 & 0.00454 \\
\hline Pu 70 & Down & 920 & No & 9.5 & 0.00633 & 0.02405 & 0.03038 & 0.02991 \\
\hline Pu 70a & Down & 920 & No. & 9.7 & 0.00452 & 0.05006 & 0.05458 & 0.05411 \\
\hline $\mathrm{Pu} 71$ & $\mathrm{Up}_{\mathrm{p}}$ & 440 & Yes & 3.0 & 0.00083 & 0.00400 & 0.00483 & 0.00436 \\
\hline $\mathrm{Pu} 71 \mathrm{a}$ & Up & 440 & Yes & 2.6 & 0.00018 & 0.00046 & 0.00064 & 0.00017 \\
\hline $\mathrm{Pu} 71 \mathrm{~b}$ & Up & 440 & Yes & 2.6 & 0.00042 & 0.00125 & 0.00168 & 0.00121 \\
\hline $\mathrm{Pu} 71 \mathrm{c}$ & Up & 440 & Yes & 2.6 & 0.00090 & 0.00026 & 0.00116 & 0.00069 \\
\hline Pu 72 & Down & 440 & Yes & 2.6 & 0.08613 & 0.56607 & 0.65220 & 0.65173 \\
\hline $\mathrm{Pu} 72 \mathrm{a}$ & Down & 440 & Yes & 2.6 & 0.07166 & 0.41948 & 0.49114 & 0.49067 \\
\hline $\mathrm{Pu} 73$ & Up & Ambient & Yes & - & 0.00081 & 0.00050 & 0.00132 & 0.00085 \\
\hline Pu 73a & Up & Ambtent & Yes & - & 0.00013 & 0 & 0.00013 & 0 \\
\hline Pu 74 & Down & Amblent & No & $=$ & 0.01986 & 0.12725 & 0.14711 & 0.14664 \\
\hline Pu $74 a$ & Down & Ambient & No & - & 0.00035 & 0.00187 & 0.00223 & 0.00176 \\
\hline $\mathrm{Pu} 74 \mathrm{~b}$ & Down & Ambient & No & - & 0.00071 & 0.00117 & 0.00187 & 0.00140 \\
\hline $\mathrm{Pu} 74 \mathrm{c}$ & Down & Amblent & No & - & 0.00088 & 0.00043 & 0.00131 & 0.00084 \\
\hline
\end{tabular}

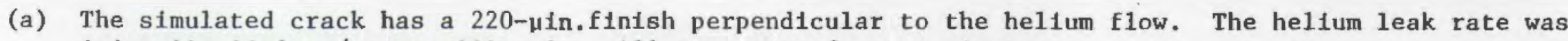
initially $13.2 \mathrm{cc} / \mathrm{sec}$ at $920 \mathrm{ps} 1 \mathrm{~g}$. All runs were for ten minutes.

(b) Based on a specific activity of $0.096 \mathrm{Cl} / \mathrm{g}$ for the $\mathrm{PuO}_{2}$ powder.

(c) The leak rate was determined by the pressure decay method at the midpoint of the run.

(d) The net total is the amount above the average containment box background of $0.00047 \mu \mathrm{g}$.

(e) Contalnment box background runs. 

TABLE A-6 SUMMARY OF ROOM TEMPERATURE PuO 2 LEAK RATE EXPERIMENTS USING A SIMULATED CRACK
WITH AN INITIAL He FLOW RATE OF $17.3 \mathrm{cc} / \mathrm{sec}$ AT 920 psig

\begin{tabular}{|c|c|c|c|c|c|c|c|c|}
\hline \multirow[b]{2}{*}{$\begin{array}{c}\text { Run } \\
\text { Number }\end{array}$} & \multirow[b]{2}{*}{$\begin{array}{c}\text { Tube } \\
\text { Position }\end{array}$} & \multirow{2}{*}{$\begin{array}{l}\text { Helium } \\
\text { Pressure, } \\
\text { psig }\end{array}$} & \multirow[b]{2}{*}{ Vibration } & \multirow{2}{*}{$\begin{array}{l}\text { Hellum } \\
\text { Leak } \\
\text { Rate, (c) } \\
\text { cc/sec }\end{array}$} & \multicolumn{2}{|c|}{ Quantity of $\mathrm{PuO}_{2}$} & \multicolumn{2}{|c|}{ Detected, $\mu g^{(b)}$} \\
\hline & & & & & $\begin{array}{r}\text { Inlet } \\
\text { Nozzle }\end{array}$ & $\begin{array}{r}\text { Final } \\
\text { Filter }\end{array}$ & Tota1 & $\begin{array}{l}\text { Net } \\
\text { Total }\end{array}$ \\
\hline B $54($ e) & Sideways & - & -- & -- & 0.00050 & 0.00040 & 0.00090 & - \\
\hline B $55^{(\mathrm{e})}$ & Sideways & -- & - & -- & 0 & 0.00104 & 0.00104 & - \\
\hline $\mathrm{Pu} 75$ & Up & 920 & No & 15.8 & 0.00096 & 0.05731 & 0.05827 & 0.05737 \\
\hline $\mathrm{Pu} 75 \mathrm{a}$ & Up & 920 & No & 16.3 & 0.00202 & 0.02667 & 0.02868 & 0.02778 \\
\hline $\mathrm{Pu} 76$ & Up & 920 & Yes & 15.8 & 0.01367 & 0.18926 & 0.20293 & 0.20203 \\
\hline $\mathrm{Pu} 76 \mathrm{a}$ & Up & 920 & Yes & 16.3 & 0.00128 & 0.00933 & 0.01061 & 0.00971 \\
\hline $\mathrm{Pu} 76 \mathrm{~b}$ & Up & 920 & Yes & 8.25 & 0.00002 & 0.01363 & 0.01365 & 0.01261 \\
\hline $\mathrm{Pu} 76 \mathrm{c}$ & Up & 920 & Yes & 8.25 & 0.00093 & 0.00374 & 0.00467 & 0.00363 \\
\hline Pu 77 & Down & 920 & No & 16.3 & 0.00424 & 0.02171 & 0.02595 & 0.02505 \\
\hline $\mathrm{Pu} 77 \mathrm{a}$ & Down & 920 & No & 16.3 & 0.00398 & 0.01969 & 0.02367 & 0.02277 \\
\hline Pu 78 & $\mathrm{Up}$ & 440 & Yes & 2.6 & 0.00030 & 0.00548 & 0.00578 & 0.00474 \\
\hline Pu 78a & Up & 440 & Yes & 2.6 & 0.00097 & 0.00553 & 0.00651 & 0.00547 \\
\hline Pu 79 & Down & 440 & Yes & 2.6 & 0.10042 & 0.35852 & 0.45894 & 0.45790 \\
\hline Pu 79a & Down & 440 & Yes & 2.6 & 0.12144 & 0.52779 & 0.64923 & 0.64819 \\
\hline $\mathrm{Pu} 80$ & $\mathrm{Up}$ & Amblent & Yes & -- & 0.00096 & 0.00036 & 0.00133 & 0.00029 \\
\hline $\mathrm{Pu} 80 \mathrm{a}$ & $\mathrm{Up}$ & Ambient & Yes & -- & 0.00076 & 0.00081 & 0.00157 & 0.00053 \\
\hline $\mathrm{Pu} 81$ & Down & Ambient & No & -- & 0.00314 & 0.01456 & 0.01770 & 0.01666 \\
\hline Pu 81a & Down & Amblent & No & -- & 0.00035 & 0.00346 & 0.00381 & 0.00277 \\
\hline
\end{tabular}

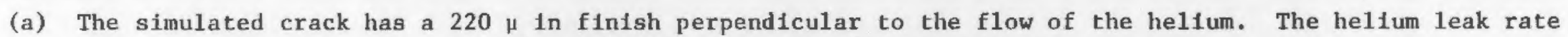
was inftially $17.3 \mathrm{cc} / \mathrm{sec}$ at $920 \mathrm{psig}$. Al1 runs were for ten minutes.

(b) Based on a speciflc activity of $0.096 \mathrm{cl} / \mathrm{g}$ for the PuO powder.

(c) The leak rate was determined by the pressure decay method at the midpoint of the run.

(d) The net total is the amount above the containment box background.

(e) Containment box background runs. B54 was used for runs Pu 75-76a and Pu 77-77a. B55 was used for. a1I other runs. 
TABLE A-7 PARAMETRIC MATRIX OF BCL PuO 2 EXPERTMENTS USING SIMULATED CRACK

\begin{tabular}{|c|c|c|c|c|c|c|c|}
\hline \multirow[b]{2}{*}{$\begin{array}{c}\text { Leak Rate (a), } \\
\mathrm{cc} / \mathrm{sec}\end{array}$} & \multirow{2}{*}{$\begin{array}{l}\text { Position: } \\
\text { Pressure: }\end{array}$} & \multicolumn{3}{|c|}{ Up } & \multicolumn{3}{|c|}{ Down } \\
\hline & & Ambient & $\begin{array}{l}440 \\
p s 1 g\end{array}$ & $\begin{array}{l}920 \\
\text { psig }\end{array}$ & Ambient & $\begin{array}{l}440 \\
\text { ps1g }\end{array}$ & $\begin{array}{l}920 \\
\text { psig }\end{array}$ \\
\hline
\end{tabular}

With Vibration
9.8
11.4
11.6
13.2
17.3

$\begin{array}{lll}2 & 3 & 2 \\ 2 & 3 & 2 \\ 2 & 2 & 4 \\ 2 & 4 & 2 \\ 2 & 2 & 4\end{array}$

$\begin{array}{lll}2 & 2 & - \\ - & 2 & - \\ - & 4 & - \\ - & 2 & - \\ - & 2 & -\end{array}$

W1thout Vibration

$\begin{array}{lllllll}9.8 & - & - & 2 & 2 & - & 2 \\ 11.4 & - & - & 2 & 2 & - & 2 \\ 11.6 & - & - & 4 & 2 & - & 6 \\ 13.2 & - & - & 4 & 4 & - & 2 \\ 17.3 & - & - & 2 & 2 & - & 2\end{array}$

TOTAL EXPERTMENTS $=92$

(a) Leak rate at 920 psig using pressure decay method; determined prior to runs. 
TABLE A-8 ARITHMETIC MEAN VALUE OF $\mathrm{PuO}_{2}$ POWDER LEAKED AND THE STANDARD DEVIATION FOR EACH EXPERIMENTAL CONDITION USING THE SIMULATED CRACK CONFIGURATION*

(Mean/Standard Deviation)

\begin{tabular}{|c|c|c|c|c|c|c|c|}
\hline \multirow[b]{2}{*}{$\begin{array}{c}\text { Leak Rate, } \\
\mathrm{cc} / \mathrm{sec}\end{array}$} & \multirow{2}{*}{$\begin{array}{l}\text { Position: } \\
\text { Pressure: }\end{array}$} & \multicolumn{3}{|c|}{ Up } & \multicolumn{3}{|c|}{ Down } \\
\hline & & Ambient & $\begin{array}{l}440 \\
\text { ps1g }\end{array}$ & $\begin{array}{r}920 \\
\text { ps1g }\end{array}$ & Ambient & $\begin{array}{r}440 \\
\text { psig }\end{array}$ & $\begin{array}{r}920 \\
\text { psig }\end{array}$ \\
\hline \multicolumn{8}{|l|}{ With Vibration } \\
\hline 9.8 & & $2 / 3$ & $1 / 1$ & $4 / 2$ & $0 / 0$ & $65 / 44$ & - \\
\hline 11.4 & & $1 / 0$ & $0 / 0$ & $0 / 0$ & - & $0 / 0$ & - \\
\hline 11.6 & & $0 / 0$ & $0 / 1$ & $441 / 872$ & - & $469 / 853$ & - \\
\hline 13.2 & & $0 / 1$ & $2 / 2$ & $6 / 2$ & - & $571 / 114$ & - \\
\hline 17.3 & & $0 / 0$ & $5 / 1$ & $57 / 97$ & - & $553 / 134$ & - \\
\hline \multicolumn{8}{|c|}{ Without Vibration } \\
\hline 9.8 & & - & - & $3 / 0$ & $0 / 0$ & - & $1 / 1$ \\
\hline 11.4 & & - & - & $0 / 0$ & $0 / 0$ & - & $0 / 0$ \\
\hline 11.6 & & - & - & $180 / 265$ & $1 / 1$ & - & $5955 / 14124$ \\
\hline 13.2 & & - & - & $29 / 50$ & $38 / 73$ & - & $42 / 17$ \\
\hline 17.3 & & - & - & $43 / 21$ & $10 / 10$ & - & $24 / 2$ \\
\hline
\end{tabular}

* Mean and standard deviation in nearest ng. 
APPENDIX B 


\section{APPENDIX B}

\section{Calculation of Time Required to Depressurize the APLA Vessel}

The volume flow rate through an orifice can be written ${ }^{[2]}$ :

$$
q_{a}=\alpha A_{\psi} \frac{p}{\rho_{a}} \sqrt{\frac{2}{R T}}
$$

where

$q_{a}=$ volume flow rate at ambient conditions

$\alpha=$ orifice coefficient

$A=$ geometric cross section of orifice

$\psi=$ dependence of flow on ratio of specific heats and pressure ratio $(0.484)$

$P=$ upstream pressure

$\rho_{\mathrm{a}}=$ ambient air density

$R=$ gas constant for air, $2.87 \times 10^{6} \frac{\mathrm{cm}^{2}}{{ }^{\circ} \mathrm{K} \mathrm{sec}}$

$\mathrm{T}=$ temperature, ${ }^{\circ} \mathrm{K}$

The rate of change of mass in the container is:

$$
\frac{d M}{d t}=-m r
$$

where

$$
\begin{aligned}
& M=\text { mass in the vessel } \\
& m_{r}=\text { mass flow rate out of the vessel }
\end{aligned}
$$

A1so:

$$
M=\rho_{\mathrm{a}} V=\frac{P V}{R T}
$$

where $V$ is the volume of the vessel, and

$$
\begin{gathered}
m_{r}=\rho_{a} q_{a} \\
\therefore \quad \frac{d}{d t}\left(\frac{P V}{R T}\right)=-\rho_{a} \propto A_{\psi} \frac{p}{\rho_{a}} \sqrt{\frac{2}{R T}}
\end{gathered}
$$

The only variable inside the pressure vessel, which is a function of time, is the pressure. Therefore, rearranging terms: 


$$
\frac{1}{P} \frac{d P}{d t}=-K
$$

where:

$$
\begin{aligned}
& K=\frac{\alpha \pi D^{2}}{4 V} \psi \sqrt{2 R T} \\
& D \text { is the orifice diameter }
\end{aligned}
$$

Integrating the above equation

one obtains

$$
\int_{P_{i}}^{P_{f}} \frac{d P}{P}=-k \int_{0}^{t_{f}} d t
$$

$$
\frac{P_{f}}{P_{i}}=e^{-x t_{f}}
$$

where the subscripts $f$ denote the final values and $i$ the initial values.

Solving for $t_{f}$ one can determine the time it takes to reach any particular pressure.

$$
t_{f}=\frac{1}{K} \ln \left(\frac{P_{i}}{P_{f}}\right)
$$

It should be remembered that this time is based on the assumption that everything is a constant except the pressure. In particular, the exit flow function, $\psi$, is taken at $i$ ts maximum value in the sample calculation given. 


\section{DISTRIBUTION}

No. of

Copies

OFF-SITE

1 DOE, Chicago Patent Group 9500 S. Cass Ave. Argonne, I11. 60439

12 $\frac{\text { NRC Division of Research }}{\text { Washington, D.C. } 20555}$

J.J. Davis

W. Lahs (10)

Frank Swanberg, Jr.

3

2

2

5

NRC Office of Nuclear Materials Safety \& Safeguards, Transportation Branch Washington, D.C. 20555

Charles E. MacDonald

C. Ross Chappel1

William H. Lake

NRC Division of Technical Information and Document Control

Washington, D.C. 20555

Sandia Laboratories Albuquerque, N.M. 87115

John A. Andersen

J.K. Cole

Battelle Memorial Institute 505 King Avenue

Columbus, Ohio 43201

W.J. Madia

E.W. Schmidt

J.D. Yesso (3)

\section{ON-SITE}

26

Pacific Northwest Laboratory
No. of

Copies

\section{1}

NRC Division of Standards Washington, D.C. 20555

Donald R. Hopkins

H.J. Bomelburg

R.L. Conley

J.W. Johnstone

J. Mishima

P.C. Owzarski

L.C. Schwendiman (10)

C.L. Simpson

Publishing Coordination (2)

Technical Information (5)

T.J. Bander

P.R. Partch

S.L. Sutter 
